\title{
MEAN-FIELD OPTIMAL CONTROL
}

\author{
Massimo Fornasier ${ }^{1}$ And Francesco Solombrino ${ }^{1}$
}

\begin{abstract}
We introduce the concept of mean-field optimal control which is the rigorous limit process connecting finite dimensional optimal control problems with ODE constraints modeling multi-agent interactions to an infinite dimensional optimal control problem with a constraint given by a PDE of Vlasov-type, governing the dynamics of the probability distribution of interacting agents. While in the classical mean-field theory one studies the behavior of a large number of small individuals freely interacting with each other, by simplifying the effect of all the other individuals on any given individual by a single averaged effect, we address the situation where the individuals are actually influenced also by an external policy maker, and we propagate its effect for the number $N$ of individuals going to infinity. On the one hand, from a modeling point of view, we take into account also that the policy maker is constrained to act according to optimal strategies promoting its most parsimonious interaction with the group of individuals. This will be realized by considering cost functionals including $L^{1}$-norm terms penalizing a broadly distributed control of the group, while promoting its sparsity. On the other hand, from the analysis point of view, and for the sake of generality, we consider broader classes of convex control penalizations. In order to develop this new concept of limit rigorously, we need to carefully combine the classical concept of mean-field limit, connecting the finite dimensional system of ODE describing the dynamics of each individual of the group to the PDE describing the dynamics of the respective probability distribution, with the well-known concept of $\Gamma$-convergence to show that optimal strategies for the finite dimensional problems converge to optimal strategies of the infinite dimensional problem.
\end{abstract}

Mathematics Subject Classification. 49J15, 49J20, 35Q83, 35Q91, 37N25.

Received June 25, 2013. Revised December 20, 2013.

Published online August 8, 2014.

\section{INTRODUCTION}

Recently there has been a strong development of literature in applied mathematics and physics describing collective behavior of multiagent systems [25-27,34,37,38,59], towards modeling phenomena in biology, such as cell aggregation and motion $[9,39,40,50]$, animal motion [5,13,16,20,21,23,27,43,46,47,54,58,64], human $[24,29,55]$ and synthetic agent behavior and interactions, such as cooperative robots $[17,42,48,57]$. As it is very hard to be exhaustive in accounting all the developments of this very fast growing field, we refer to $[12,14,60]$ for recent surveys.

Keywords and phrases. Sparse optimal control, mean-field limit, $\Gamma$-limit, optimal control with ODE constraints, optimal control with PDE constraints.

1 Technische Universität München, Fakultät Mathematik, 385748 Boltzmannstrasse, Garching bei München, Germany.

massimo.fornasier@ma.tum.de; francesco.solombrino@ma.tum.de 
Most of these models start from particle-like systems, borrowing a leaf from Newtonian physics, by including fundamental "social interaction" forces, such as attraction, repulsion, self-drive, orientation and alignment etc. within classical systems of 2 nd order equations, governing the evolution of the status, usually the spatial motion, of each agent. One fundamental goal of these studies is to clarify the relationship between the interplay of such simple binary forces, being the "first principles" of social interaction, and the potential emergence of a global behavior in the form of specific patterns, as the result of the re-iterated superposition in time and group-wise of such forces. For patterns we do not necessarily mean steady states, as one considers in the study of crystalline structures, but rather structured evolutions, such as the formation of flocks or swarms in animal motion. Due to their discrete nature and the direct description of the dynamics in terms of the single agent, such particle models are also called Individual Based Models. While in some cases, for instance in flocking models [27,28], it is possible to describe rather precisely the mechanism of pattern formation, for most of the models the description of the asymptotic behavior of a very large system of particles can become an impossible task. A classical way to approach the global description of the system is then to focus on its mean behavior, as in the classical mean-field theory one studies the evolution of a large number of small individuals freely interacting with each other, by simplifying the effect of all the other individuals on any given individual by a single averaged effect. This results in considering the evolution of the particle density distribution in the state variables, leading to so-called mean-field partial differential equations of Vlasov- or Boltzmann-type [49]. We refer to [12] and the references therein for a recent survey on some of the most relevant mathematical aspects on this approach to swarm models.

On the one hand, in certain circumstances, the formation of a specific pattern is conditional to the initial datum, being positioned in a corresponding basin of attraction, which sometimes can be characterized, see [16] for an interesting example of modeling of possible multiple patterns. On the other hand, the choice of the initial condition outside such a basin of attraction does not give in general any guarantee of stable pattern formation. Thus it is interesting to question whether an "external player" or "policy maker" can intervene on the system towards pattern formation, also in those situations where this phenomenon is not the result of independent self-organization. The intervention may be modeled as an additional control vector field subjected to certain bounds, representing the limitations (in terms of resources, strength etc.) of the external policy maker.

In the recent work [11] the authors investigated, specifically for individual based flocking models of Cucker-Smale type $[27,28]$, how sparse controls can be applied in order to always ensure pattern formation, in this case the emergence of consensus.

For sparse control we mean that the policy maker intervenes the minimal amount of times on the minimal amount of individual agents. Surprisingly this control strategy turns out not only to be economical in terms of interactions between the policy maker and the group of agents, but also in terms of enhancing the rate of convergence to pattern formation.

While the work [11] clarified the basis of sparse stabilization and optimal control for individual based models, so far it has not been explored how such concepts could be rigorously connected, through a proper limit process for the number $\mathrm{N}$ of agents going to infinity, to continuum models, as in the classical aforementioned mean-field theory of uncontrolled systems.

In this paper we want to pose the foundations of the discrete/finite dimensional-continuum/infinite dimensional limit for $N \rightarrow \infty$ of ODE constrained control problems of the type:

$$
\left\{\begin{array}{l}
\dot{x}_{i}=v_{i}, \\
\dot{v}_{i}=\left(H \star \mu_{N}\right)\left(x_{i}, v_{i}\right)+f\left(t, x_{i}, v_{i}\right), i=1, \ldots N, \quad t \in[0, T],
\end{array}\right.
$$

where

$$
\mu_{N}=\frac{1}{N} \sum_{j=1}^{N} \delta_{\left(x_{i}, v_{i}\right)}
$$


is the empirical atomic measure supported on the agents states $\left(x_{i}, v_{i}\right) \in \mathbb{R}^{2 d}$, controlled by the minimizer of the cost functional

$$
\mathcal{E}_{\psi}^{N}(f):=\int_{0}^{T} \int_{\mathbb{R}^{2 d}}\left(L\left(x, v, \mu_{N}(t)\right)+\psi(f(t, x, v)) \mathrm{d} \mu_{N}(t)(x, v) \mathrm{d} t\right.
$$

where $\psi: \mathbb{R}^{d} \rightarrow[0,+\infty)$ is a nonnegative convex function, $f(t, x, v): \mathbb{R} \times \mathbb{R}^{d} \times \mathbb{R}^{d} \rightarrow \mathbb{R}^{d}$ is a Carathéodory function, being an absolutely continuous control vector field with a certain sublinear growth in the variables $x$ and $v, H: \mathbb{R}^{2 d} \rightarrow \mathbb{R}^{d}$ is a sublinear and locally Lipschitz continuous interaction kernel, and $L: \mathbb{R}^{2 d} \times \mathcal{P}_{1}\left(\mathbb{R}^{2 d}\right) \rightarrow \mathbb{R}_{+}$ is a continuous function with respect to the product topology generated by the Euclidean distance on $\mathbb{R}^{2 d}$ and the Wasserstein distance $\mathcal{W}_{1}$ on $\mathcal{P}_{1}\left(\mathbb{R}^{2 d}\right)$. Let us stress that the optimal control problem (1.1) and (1.2) is actually nonstandard and its well-posedness does not follow from classical existence arguments such as in ([7], Chapter 5). We shall prove the existence of controls for (1.1) and (1.2) in Theorem 3.3, based on the compactness arguments derived in Section 2 for the considered class of feedback control functions. In fact the novelty with respect to usual closed loop control problems stems precisely from the feedback form of the control in terms of a locally Lipschitz continuous function $f(t, \cdot, \cdot)$ of the state variables $\left(x_{j}, v_{j}\right)$, for $j=1, \ldots, N$. In order to grasp, although only intuitively, this fundamental difference, let us mention that a relevant choice for $\psi$, modeling a situation of particular interest, is $\psi(\cdot)=\gamma|\cdot|$, for $\gamma>0$. In this case the minimization of $\mathcal{E}_{\psi}^{N}$ simultaneously promotes a choice of an optimal control $f$, which instantaneously steers the system in the direction of the basin of attraction as a consequence of the minimization of the term involving $L$, and the sparsity of $f$ by means of the $L^{1}$-norm penalization term

$$
\int_{\mathbb{R}^{2 d}}|f(t, x, v)| \mathrm{d} \mu_{N}(x, v)=\frac{1}{N} \sum_{j=1}^{N}\left|f\left(t, x_{j}, v_{j}\right)\right|
$$

With this we mean that $\operatorname{supp}(f(t, \cdot)) \cap \operatorname{supp}\left(\mu_{N}\right)$ is actually expected to be a "small set". The use of (scalar) $\ell^{1}$-norms to penalize controls dates back to the 60 's with the models of linear fuel consumption [22]. More recent work in dynamical systems [62] resumes again $\ell^{1}$-minimization emphasizing its sparsifying power. Also in optimal control with partial differential equation constraints it became rather popular to use $L^{1}$-minimization to enforce sparsity of controls $[15,18,19,35,51,56,63]$, for instance in the modeling of optimal placing of actuators or sensors. Let us also mention the recent related work [52] on the optimal design problem for sparse control of wave equations, where a suitable convex relaxation (see [52], Rem. 1) leads to the transformation of the problem based on an optimization over characteristic functions of small sets to a problem of minimization over terms of the type (1.3).

Now, if we were considering the identification $u_{j}=f\left(t, x_{j}, v_{j}\right)$ and we were solving a standard optimal control problem for the non-feedback controls $u(t)=\left(u_{1}(t), \ldots, u_{N}(t)\right)$, in view of the sparsity promoted by (1.3), we could easily end up in situations where $\left|u_{j}(t)\right|=M \in \mathbb{R}_{+}$and $\left|u_{j^{\prime}}(t)\right|=0$ for $j \neq j^{\prime}$ and $\left(x_{j}(t), v_{j}(t)\right)$ arbitrarily close to $\left(x_{j^{\prime}}(t), v_{j^{\prime}}(t)\right)$, hence violating the assumed Lipschitz continuity of any $f$ for which $u_{j}(t)=f\left(t, x_{j}, v_{j}\right)$ and $u_{j^{\prime}}(t)=f\left(t, x_{j^{\prime}}, v_{j^{\prime}}\right)$. As we will see below, the Lipschitz continuity of a function $f$ cannot be easily relaxed as an assumption as soon as we wish to consider it eventually as a control for a partial differential equation of the type (1.6) below.

The general system (1.1) includes, for instance, the sparsely controlled Cucker-Smale type of models of flocking [11], obtained by choosing $H(x, v)=a(|x|) v$, where $a \in C^{1}([0,+\infty))$ is a nonincreasing positive function, and $L\left(x, v, \mu_{N}\right)=\left|v-\left(\int_{\mathbb{R}^{2 d}} w \mathrm{~d} \mu_{N}(y, w)\right)\right|^{2}=\left|v-\frac{1}{N} \sum_{j=1}^{N} v_{j}\right|^{2}$, for which one gets

$$
\left\{\begin{array}{l}
\dot{x}_{i}=v_{i}, \\
\dot{v}_{i}=\frac{1}{N} \sum_{j=1}^{N} a\left(\left|x_{j}-x_{i}\right|\right)\left(v_{j}-v_{i}\right)+f\left(t, x_{i}, v_{i}\right), i=1, \ldots N, \quad t \in[0, T],
\end{array}\right.
$$


subjected to the optimal control $f$ minimizing the cost functional

$$
\mathcal{E}_{\gamma}^{N}(f):=\int_{0}^{T} \frac{1}{N} \sum_{i=1}^{N}\left(\left|v_{i}-\frac{1}{N} \sum_{j=1}^{N} v_{j}\right|^{2}+\gamma\left|f\left(t, x_{i}, v_{i}\right)\right|\right) \mathrm{d} t .
$$

The interested reader can compare (1.4) and (1.5) with the more classically formulated sparse optimal control problem studied in ([11], Sect. 5).

The main result of this work is to clarify in which sense the finite dimensional solutions of (1.1) and (1.2) converges for $N \rightarrow \infty$ to a solution of the PDE constrained problem

$$
\frac{\partial \mu}{\partial t}+v \cdot \nabla_{x} \mu=\nabla_{v} \cdot[(H \star \mu+f) \mu]
$$

controlled by the minimizer $f$ of the cost functional

$$
\mathcal{E}_{\psi}(f):=\int_{0}^{T} \int_{\mathbb{R}^{2 d}}(L(x, v, \mu(t))+\psi(f(t, x, v))) \mathrm{d} \mu(t)(x, v) \mathrm{d} t,
$$

where $\mu:[0, T] \rightarrow \mathcal{P}_{1}\left(\mathbb{R}^{2 d}\right)$ is a probability measure valued weak solution to (1.6). Our arguments will be based on the combination of the concepts of mean-field limit, using techniques of optimal transport [3], in order to connect (1.1) to (1.6), and $\Gamma$-limit [30] in order to connect the minimizations of (1.2) and (1.7). Accordingly we call this limit process mean-field optimal control.

Let us stress that some of the relevant ingredients of our theory were already partially available in the literature. In particular, the rigorous derivation of the mean-field limits to connect (1.1) to (1.6) for situations where no control is addressed, i.e., when $f \equiv 0$, has been already considered, for instance, in [10]. Nevertheless, although it represents a minor extension, the situation where a control $f$ is present in the equations, and it has potentially a discontinuous nature in time, requires to generalize the results in [10] to solutions of Carathéodory for (1.1) [32]. We sketch these generalizations in the Appendix for the sake of completeness. In particular, existence, uniqueness, and stability of weak measure-valued solutions to (1.6) with compactly supported data will be given in details in Theorems 4.7 and A.8.

Additional tools are certain compactness arguments in $L^{q}\left((0, T), W_{l o c}^{1, \infty}\left(\mathbb{R}^{2 d}, \mathbb{R}^{d}\right)\right)$ for the derivation of a limit for the controls (Thm. 2.10 and Cor. 2.11), and compactness arguments in 1-Wasserstein distance for probability measures in $\mathcal{P}_{1}\left(\mathbb{R}^{2 d}\right)$ in order to derive limits of the empirical measures to weak solutions of (1.6). Finally, the optimality conditions for the limit controls will be derived using lower-semicontinuity arguments for the energy $\mathcal{E}_{\psi}(f)$ in order to obtain the $\Gamma$-lim inf condition (Thm. 4.4), and the construction of solutions to (1.6) in order to define a recovery sequence for the $\Gamma$-lim sup condition (Thm. 4.7).

Beside the specific novelty of our model, where we considered collective behavior (sparsely) controlled by an external policy maker restricted by limited resources, we stress again that the originality of our analysis stands precisely in the combination of the concepts of mean-field- and $\Gamma$-limits, where the reference topologies are those of $\mathcal{P}_{1}\left(\mathbb{R}^{2 d}\right)$ for the solutions and $L^{q}\left((0, T), W_{\text {loc }}^{1, \infty}\left(\mathbb{R}^{2 d}, \mathbb{R}^{d}\right)\right)$ for the controls. This distinguishes our work from other conceptually similar approaches where limits of finite dimensional optimal control problems to infinite dimensional control problems are considered. We refer in particular to two main directions.

The first is the discretization of PDE constrained optimal control problems by means, e.g., of finite element methods. One defines a suitable finite dimensional time and/or space discretization and shows that corresponding finite dimensional optimal control solutions converge to the solution of the PDE constrained optimal control problem. Let us stress that such a type of arguments have been applied mainly for elliptic and parabolic type of equations, and the tools used are either explicit a priori Galerkin-type error estimates or adaptive discretizations, driven by a posteriori error estimates in classical Sobolev spaces. Without being able to be at all exhaustive in describing the vast literature on this well-established methodology, we refer to a classical reference [33] and to the recent survey paper [53] and the bibliography therein. 
In order to encounter transport equations, one needs to refer to the second main direction conceptually similar to our approach, i.e., the mean-field games, introduced by Lasry and Lions [41], see in particular ([41], Sect. 2.6), and independently in the optimal control community under the name Nash Certainty Equivalence (NCE) within the work [36], later greatly popularized within consensus problems, for instance in [44,45]. The first fundamental difference with our work is that in (mean-field) games, each individual agent is competing freely with the others towards the optimization of its individual goal, as for instance in the financial market, whereas in our model we are concerned with the optimization of the intervention of an external policy maker or coordinator endowed with rather limited resources to help the system to form a pattern, when self-organization does not realize it autonomously, as it is a case, e.g., in modeling economical policies and government strategies. Let us stress that in our model we are particularly interested to sparsify the control towards most effective results, and also that such an economical concept does not appear anywhere in the literature when we deal with mean-field limits of large particle systems. Secondly in mean-field games the stochastic component plays a relevant role (also for the technical derivation of mean-field limits), while in our deterministic model no stochastic terms are necessarily requested in order to have sufficient regularization for deriving rigorously the mean-field limit.

At this point it is also very important to mention the concept of mean-field type control independently developed recently by Bensoussan, Frehse, and Yam [6], and addressing similar optimal control problems as (1.6) and (1.7) in a stochastic setting, with strong emphasis on the actual computation of first order optimality equations characterizing the controls. We also mention the earlier results of this type $[1,4,8]$. Differently from these papers, our results focus on the rigorous derivation and the well-posedness of (1.6) and (1.7) from the individual based model (1.1) and (1.2), combining techniques of optimal transport and variational calculus in a fully deterministic setting.

The paper is organized as follows: in Section 2 we introduce the class of control functions and we prove its closedness and compactness properties, and certain lower-semicontinuity results related to the cost functional (1.7). Section 3 is dedicated to the finite dimensional optimal control problem (1.1) and (1.2) and its well-posedness. In Section 4 we address both the mean-field limit to connect (1.1) to (1.6) and the conditions of $\Gamma$-convergence to connect the minimizations of (1.2) and (1.7), to eventually conclude with Section 5 where we state our main mean-field optimal control result, which summarizes all our findings. For the sake of a broad readability of the paper and its self-containedness we also included an Appendix recalling the relevant results on Carathéodory solutions of ODEs and how they are related via the method of the characteristics to solutions of (1.6).

\section{THE SPACE OF ADMISSIBLE CONTROLS}

\subsection{Admissible controls}

Let $d \geq 1$ be the dimensionality of the control output, $n \geq 1$ be the dimensionality of the state variables (later we will consider $n=2 d$ ).

Definition 2.1. For a horizon time $T>0$, and an exponent $1 \leq q<+\infty$ we fix a control bound function $\ell \in L^{q}(0, T)$. The class of admissible control functions $\mathcal{F}_{\ell}([0, T])$ is so defined: $f \in \mathcal{F}_{\ell}([0, T])$ if and only if

(i) $f:[0, T] \times \mathbb{R}^{n} \rightarrow \mathbb{R}^{d}$ is a Carathéodory function,

(ii) $f(t, \cdot) \in W_{l o c}^{1, \infty}\left(\mathbb{R}^{n}, \mathbb{R}^{d}\right)$ for almost every $t \in[0, T]$, and

(iii) $|f(t, 0)|+\operatorname{Lip}\left(f(t, \cdot), \mathbb{R}^{d}\right) \leq \ell(t)$ for almost every $t \in[0, T]$.

Functions in the class $\mathcal{F}_{\ell}([0, T])$ can be also regarded as measurable mappings with values in Banach spaces, as we clarify in the next two remarks.

Remark 2.2. Every control function $f \in \mathcal{F}_{\ell}([0, T])$ can be identified with a mapping $f:[0, T] \rightarrow W_{\text {loc }}^{1, \infty}\left(\mathbb{R}^{n} ; \mathbb{R}^{d}\right)$ where $f(t)$ is simply the function taking the value $f(t, x)$ at $x$. Let us show now that $f \in L^{q}\left((0, T), W^{1, p}\left(\Omega, \mathbb{R}^{d}\right)\right)$ for every open bounded subset $\Omega \subset \mathbb{R}^{n}$ and $1<p<+\infty$, with $q$ being exactly the integrability exponent of $\ell$. 
To prove that the mapping is measurable, by separability of $W^{1, p}\left(\Omega, \mathbb{R}^{d}\right)$, it suffices to show weak measurability. Since $f$ is Carathéodory, by density of atomic measures in the weak-* topology of measures, the map $t \rightarrow\langle f(t), \mu\rangle$ is measurable for every $\mu \in M_{b}\left(\Omega, \mathbb{R}^{d}\right)$. The former duality pairing is the standard one between continuous functions and measures. This holds now in particular when $\mu$ is a function in $L^{\left(p^{*}\right)^{\prime}}\left(\Omega, \mathbb{R}^{d}\right)$, with $p^{*}$ the Sobolev exponent. Since $W^{1, p}\left(\Omega, \mathbb{R}^{d}\right)$ is densely embedded into $L^{p^{*}}\left(\Omega, \mathbb{R}^{d}\right)$, then $L^{\left(p^{*}\right)^{\prime}}\left(\Omega, \mathbb{R}^{d}\right)$ is densely embedded into the dual space of $W^{1, p}\left(\Omega, \mathbb{R}^{d}\right)$ endowed with the weak topology. It follows that $t \rightarrow\langle\phi, f(t)\rangle$ is measurable for all $\phi \in\left(W^{1, p}\left(\Omega, \mathbb{R}^{d}\right)\right)^{\prime}$, as we wanted. Finally, one easily has by the assumptions that $\|f(t)\|_{W^{1, p}\left(\Omega, \mathbb{R}^{d}\right)} \in L^{q}(0, T)$, so that $f \in L^{q}\left((0, T), W^{1, p}\left(\Omega, \mathbb{R}^{d}\right)\right)$.

Remark 2.3. Conversely, consider a mapping $f:[0, T] \rightarrow W_{l o c}^{1, \infty}\left(\mathbb{R}^{n}, \mathbb{R}^{d}\right)$ such that $f \in L^{q}\left((0, T), W^{1, p}\left(\Omega, \mathbb{R}^{d}\right)\right)$ for every open bounded subset $\Omega \subset \mathbb{R}^{n}$ and $1<p<+\infty$, the identification $f(t, x)=f(t)(x)$ for all $x \in \Omega$ gives us a Carathéodory function. It makes then sense to consider the subset $C_{\ell, \Omega}$ of $L^{q}\left((0, T), W^{1, p}\left(\Omega, \mathbb{R}^{d}\right)\right)$ defined by

$$
C_{\ell, \Omega}:=\left\{f \in L^{q}\left((0, T), W^{1, p}\left(\Omega, \mathbb{R}^{d}\right)\right):|f(t, 0)|+\operatorname{Lip}\left(f(t, \cdot), \mathbb{R}^{d}\right) \leq \ell(t) \text { for a.e. } t \in[0, T]\right\} .
$$

It easily turns out that $C_{\ell, \Omega}$ is convex. Furthermore, if $f \in C_{\ell, \Omega}$ for all $\Omega \subset \mathbb{R}^{n}$, it can be identified with a $f \in \mathcal{F}_{\ell}([0, T])$.

In the following, functions in the class $F_{\ell}([0, T])$ will be identified with measurable mappings $f:[0, T] \rightarrow$ $W_{l o c}^{1, \infty}\left(\mathbb{R}^{n}, \mathbb{R}^{d}\right)$ and vice versa, according to Remarks 2.2 and 2.3 , without further specification.

We also point out some closedness properties of the convex set $C_{\ell, \Omega}$ introduced in (2.1).

Remark 2.4. Fix $1<p<+\infty$ and a bounded smooth subset $\Omega \subset \mathbb{R}^{n}$. Take a sequence $\left(f_{j}\right)_{j \in \mathbb{N}}$ in $C_{\ell, \Omega}$ such that $f_{j}(t)$ converges to $f(t)$ in $W^{1, p}\left(\Omega, \mathbb{R}^{d}\right)$ for a.e. $t \in[0, T]$. Then, for a.e. $t$ the $W^{1, \infty}\left(\Omega, \mathbb{R}^{d}\right)$ norm of $f_{j}(t)$ is bounded because of the definition of $C_{\ell, \Omega}$, so that $f_{j}(t)$ converges to $f(t)$ weakly-* in $W^{1, \infty}\left(\Omega, \mathbb{R}^{d}\right)$ for a.e. $t \in[0, T]$, and

$$
|f(t, 0)|+\operatorname{Lip}(f(t, \cdot), \Omega) \leq \liminf _{j \rightarrow \infty}\left|f_{j}(t, 0)\right|+\operatorname{Lip}\left(f_{j}(t, \cdot), \Omega\right) .
$$

It follows that $C_{\ell, \Omega}$ is closed with respect to pointwise a.e. convergence, and therefore in the $L^{q}\left((0, T), W^{1, p}\left(\Omega, \mathbb{R}^{d}\right)\right)$ norm topology, since any Cauchy sequence in $L^{q}\left((0, T), W^{1, p}\left(\Omega, \mathbb{R}^{d}\right)\right)$ has a pointwise a.e. converging subsequence. Since $C_{\ell, \Omega}$ is convex, we deduce from Mazur's Lemma that it is also closed in the weak topology of $L^{q}\left((0, T), W^{1, p}\left(\Omega, \mathbb{R}^{d}\right)\right)$.

In the following we shall usually fix a horizon time $T>0$ and denote $\mathcal{F}_{\ell}:=\mathcal{F}_{\ell}([0, T])$, omitting the time interval. The integrability exponent of $\ell$ will be depending on the cost functional we consider, as we will make precise in Section 3. In the case of a cost functional of the type (1.5), the one we are mainly interested in, we will choose $q=1$.

\subsection{Compactness, closedness, and lower semicontinuity properties}

The following compactness result is a sort of generalization of the Dunford-Pettis theorem [2], Theorem 1.38 for equi-integrable families of functions with values in a reflexive and separable Banach spaces. Its derivation is standard, but we include its proof for the sake of completeness.

Theorem 2.5. Let $X$ be a reflexive and separable Banach space. Let $\left(f_{j}\right)_{j \in \mathbb{N}}$ be a sequence of functions in $L^{q}((0, T), X)$ with $1 \leq q<+\infty$. Let us also assume that there exists a map $m \in L^{q}(0, T)$ such that $\left\|f_{j}(t)\right\|_{X} \leq$ $m(t)$ for almost all $t \in[0, T]$. Then there exist a subsequence $\left(f_{j_{k}}\right)_{k \in \mathbb{N}}$ and a function $f \in L^{q}((0, T), X)$ such that

$$
\lim _{k \rightarrow \infty} \int_{0}^{T}\left\langle\phi(t), f_{j_{k}}(t, \cdot)-f(t, \cdot)\right\rangle \mathrm{d} t=0
$$


for all $\phi \in L^{q^{\prime}}\left((0, T), X^{\prime}\right)$, with $q^{\prime}$ the conjugate exponent of $q$, and

$$
w-\lim _{k \rightarrow \infty} \int_{t_{1}}^{t_{2}} f_{j_{k}}(t) \mathrm{d} t=\int_{t_{1}}^{t_{2}} f(t) \mathrm{d} t, \quad \text { for all } t_{1} \cdot t_{2} \in[0, T],
$$

where the limit is in the sense of the weak topology of $X$ and the integrals are in the sense of Bochner.

Proof. Let us first of all recall that, as $X$ is reflexive, it has the Radon-Nikodym property: in particular, given $F \in \operatorname{AC}([0, T], X)$ there exists $f \in L^{1}((0, T), X)$ such that

$$
F\left(t_{2}\right)-F\left(t_{1}\right)=\int_{t_{1}}^{t_{2}} f(t) \mathrm{d} t, \quad \text { for all } t_{1}, t_{2} \in[0, T] .
$$

Let us now define $F_{j}(s)=\int_{0}^{s} f_{j}(t) \mathrm{d} t$, and we have

$$
\left\|F_{j}\left(t_{2}\right)-F_{j}\left(t_{1}\right)\right\|_{X} \leq \int_{t_{1}}^{t_{2}} m(t) \mathrm{d} t, \quad \text { for all } t_{1}, t_{2} \in[0, T] .
$$

Therefore $F_{j}$ are equi-bounded and equi-absolutely continuous, and by Ascoli-Arzelà theorem, there exist a subsequence $\left(F_{j_{k}}\right)_{k \in \mathbb{N}}$ and a function $F \in \mathrm{AC}([0, T], X)$ such that

$$
w-\lim _{k \rightarrow \infty}\left(F_{j_{k}}\left(t_{2}\right)-F_{j_{k}}\left(t_{1}\right)\right)=F\left(t_{2}\right)-F\left(t_{1}\right),
$$

weakly in $X$ for all $t_{1}, t_{2} \in[0, T]$ (this is a consequence of the separability of $X$ ). By the aforementioned Radon-Nikodym property, there exists $f \in L^{1}((0, T), X)$ such that

$$
F\left(t_{2}\right)-F\left(t_{1}\right)=\int_{t_{1}}^{t_{2}} f(t) \mathrm{d} t, \quad \text { for all } t_{1}, t_{2} \in[0, T] .
$$

Moreover, by the weak limit (2.4) and lower-semicontinuity of the norm of $X$,

$$
\left\|F\left(t_{2}\right)-F\left(t_{1}\right)\right\|_{X} \leq \liminf _{k \rightarrow \infty}\left\|F_{j_{k}}\left(t_{2}\right)-F_{j_{k}}\left(t_{1}\right)\right\|_{X} \leq \int_{t_{1}}^{t_{2}} m(t) \mathrm{d} t
$$

hence the modulus of absolute continuity of $F$ is again $\int_{t_{1}}^{t_{2}} m(t) \mathrm{d} t$ and, by the Lebsegue theorem for functions with values in Banach spaces ([31], 2.9.9), we obtain $\|f(t)\|_{X} \leq m(t)$ for almost every $t \in[0, T]$. It follows that $f \in L^{q}((0, T), X)$. As

$$
w-\lim _{k \rightarrow \infty} \int_{t_{1}}^{t_{2}} f_{j_{k}}(t) \mathrm{d} t=\int_{t_{1}}^{t_{2}} f(t) \mathrm{d} t
$$

weakly in $X$ for all $t_{1}, t_{2} \in[0, T]$, we actually have

$$
w-\lim _{k \rightarrow \infty} \int_{A} f_{j_{k}}(t) \mathrm{d} t=\int_{A} f(t) \mathrm{d} t,
$$

for all $A$ open subsets of $[0, T]$, and therefore for all Borel subsets $A$ of $[0, T]$. Hence for any simple function $\phi(t)=\sum_{i=1}^{m} \phi_{i} \chi_{A_{i}}(t)$ where $\phi_{i} \in X^{\prime}$, we have $\phi \in L^{\infty}\left((0, T), X^{\prime}\right)$ and

$$
\lim _{k \rightarrow \infty} \int_{0}^{T}\left\langle\phi(t), f_{j_{k}}(t)\right\rangle \mathrm{d} t=\int_{0}^{T}\langle\phi(t), f(t)\rangle \mathrm{d} t,
$$

where now the convergence is of real values, and one concludes the proof by density of such simple functions in $L^{q^{\prime}}\left((0, T), X^{\prime}\right)$ and an application of the dominated convergence theorem taking into account the $q$-integrability of $m$.

The following local compactness property is fundamental to our analysis. 
Theorem 2.6. Let $\Omega$ be a bounded, smooth, and open subset of $\mathbb{R}^{n}$ and let $1<p<\infty$ and $1 \leq q<+\infty$. Assume that $\left(f_{j}\right)_{j \in \mathbb{N}}$ be a sequence of functions in $L^{q}\left((0, T), W^{1, p}\left(\Omega, \mathbb{R}^{d}\right)\right)$ such that

$$
\left|f_{j}(t, 0)\right|+\operatorname{Lip}\left(f_{j}(t, \cdot), \Omega\right) \leq \ell(t) \in L^{q}(0, T), \quad \text { for almost every } t \in[0, T], \text { for all } j \in \mathbb{N} .
$$

Then there exist a subsequence $\left(f_{j_{k}}\right)_{k \in \mathbb{N}}$ and a function $f \in L^{q}\left((0, T), W^{1, p}\left(\Omega, \mathbb{R}^{d}\right)\right)$ such that

$$
w-\lim _{k \rightarrow \infty} f_{j_{k}}=f
$$

weakly in $L^{q}\left((0, T), W^{1, p}\left(\Omega, \mathbb{R}^{d}\right)\right)$, and

$$
|f(t, 0)|+\operatorname{Lip}(f(t, \cdot), \Omega) \leq \ell(t), \text { for almost every } t \in[0, T] .
$$

Proof. By an application of Theorem 2.5 for $X=W^{1, p}\left(\Omega, \mathbb{R}^{d}\right)$, there exist a subsequence $\left(f_{j_{k}}\right)_{k \in \mathbb{N}}$ and a function $f \in L^{q}\left((0, T), W^{1, p}\left(\Omega, \mathbb{R}^{d}\right)\right)$ such that (2.5) holds. It remains to show (2.6). Defining $C_{\ell, \Omega}$ as in (2.1), (2.6) is equivalent to saying that $f \in C_{\ell, \Omega}$. The conclusion is therefore immediate, since $C_{\ell, \Omega}$ is closed with respect to the weak topology $L^{q}\left((0, T), W^{1, p}\left(\Omega, \mathbb{R}^{d}\right)\right)$ by Remark 2.4 .

An immediate consequence of the previous theorem is the following weak compactness result in $\mathcal{F}_{\ell}$.

Corollary 2.7. Let $1<p<\infty$. Assume that $\left(f_{j}\right)_{j \in \mathbb{N}}$ be a sequence of functions in $\mathcal{F}_{\ell}$ for a given function $\ell \in L^{q}(0, T), 1 \leq q<+\infty$. Then there exist a subsequence $\left(f_{j_{k}}\right)_{k \in \mathbb{N}}$ and a function $f \in \mathcal{F}_{\ell}$, such that

$$
\lim _{k \rightarrow \infty} \int_{0}^{T}\left\langle\phi(t), f_{j_{k}}(t, \cdot)-f(t, \cdot)\right\rangle \mathrm{d} t=0,
$$

for all $\phi \in L^{q^{\prime}}\left([0, T], H^{-1, p^{\prime}}\left(\mathbb{R}^{n}, \mathbb{R}^{d}\right)\right)$ such that $\operatorname{supp}(\phi(t)) \Subset \Omega$ for all $t \in[0, T]$, where $\Omega$ is a relatively compact set in $\mathbb{R}^{n}$. Here the symbol $\langle\cdot, \cdot\rangle$ denotes the duality between $W^{1, p}$ and its dual $H^{-1, p^{\prime}}$.

Proof. By considering an invading countable sequence $\left(\Omega_{h}\right)_{h \in \mathbb{N}}$ of bounded, smooth, and open subsets of $\mathbb{R}^{n}$ and using a diagonal argument, one shows that there exist a subsequence $\left(f_{j_{k}}\right)_{k \in \mathbb{N}}$ and a function $f \in L^{q}\left((0, T), W^{1, p}\left(\Omega_{h}, \mathbb{R}^{d}\right)\right)$ such that $w-\lim _{k \rightarrow \infty} f_{j_{k}}=f$ weakly in $L^{q}\left((0, T), W^{1, p}\left(\Omega_{h}, \mathbb{R}^{d}\right)\right)$ for all $h \in \mathbb{N}$, and

$$
|f(t, 0)|+\operatorname{Lip}\left(f(t, \cdot), \mathbb{R}^{n}\right)=\sup _{h \in \mathbb{N}}|f(t, 0)|+\operatorname{Lip}\left(f(t, \cdot), \Omega_{h}\right) \leq \ell(t), \text { for almost every } t \in[0, T] .
$$

Hence actually $f \in \mathcal{F}_{\ell}$. In order to conclude the validity of (2.7), it is now sufficient to observe that if $\Omega$ is relatively compact, then there exists $h \in \mathbb{N}$ such that $\Omega \subset \Omega_{h}$.

Remark 2.8. By duality and Morrey's embedding theorem for $n<p<\infty$, if $\psi(t)$ is actually measure valued, then it is also $H^{-1, p^{\prime}}$-valued. This observation is used silently in the proofs of the results which follow.

Remark 2.9. The existence of a subsequence of indexes $j_{k}$ independent of $t$ so that $f_{j_{k}}(t, \cdot)$ converges to $f(t, \cdot)$ weakly in $W_{l o c}^{1, p}\left(\mathbb{R}^{n}, \mathbb{R}^{d}\right)$ for all $t \in[0, T]$ is in general false. It suffices to think of the sequence $f_{j}(t, \zeta)=\sin (2 \pi j t) \zeta$ which, however, converges to 0 in the sense of (2.7) as a consequence of the Riemann-Lebesgue Lemma.

In the following we consider the space $\mathcal{P}_{1}\left(\mathbb{R}^{n}\right)$, consisting of all probability measures on $\mathbb{R}^{n}$ with finite first moment. On this set we shall consider the following distance, called the Monge-Kantorovich-Rubistein distance,

$$
\mathcal{W}_{1}(\mu, \nu)=\sup \left\{\left|\int_{\mathbb{R}^{n}} \varphi(x) \mathrm{d}(\mu-\nu)(x)\right|: \varphi \in \operatorname{Lip}\left(\mathbb{R}^{n}\right), \quad \operatorname{Lip}(\varphi) \leq 1\right\},
$$

where $\operatorname{Lip}\left(\mathbb{R}^{n}\right)$ is the space of Lipschitz continuous functions on $\mathbb{R}^{n}$ and $\operatorname{Lip}(\varphi)$ the Lipschitz constant of a function $\varphi$. Such a distance can also be represented in terms of optimal transport plans by Kantorovich duality 
in the following manner: if we denote $\Pi(\mu, \nu)$ the set of transference plans between the probability measures $\mu$ and $\nu$, i.e., the set of probability measures on $\mathbb{R}^{n} \times \mathbb{R}^{n}$ with first and second marginals equal to $\mu$ and $\nu$ respectively, then we have

$$
\mathcal{W}_{1}(\mu, \nu)=\inf _{\pi \in \Pi(\mu, \nu)}\left\{\int_{\mathbb{R}^{n} \times \mathbb{R}^{n}}|x-y| \mathrm{d} \pi(x, y)\right\} .
$$

In the form $(2.9)$ the distance $\mathcal{W}_{1}$ is also known as the 1 -Wasserstein distance. We refer to [3,61] for more details.

Theorem 2.10. For a given $\ell \in L^{1}(0, T)$, let $\left(f_{k}\right)_{k \in \mathbb{N}}$ be a sequence of functions in $\mathcal{F}_{\ell}$ converging to $f$ in the sense of (2.7). Let $\mu_{k}:[0, T] \rightarrow \mathcal{P}_{1}\left(\mathbb{R}^{n}\right)$ be a sequence of functions taking values in the probability measures with finite first moment, and $\mu:[0, T] \rightarrow \mathcal{P}_{1}\left(\mathbb{R}^{n}\right)$ such that

$$
\sup _{t \in[0, T]} \int_{\mathbb{R}^{n}}|x| \mathrm{d} \mu_{k}(t, x)=M<\infty,
$$

and

$$
\lim _{j} \mathcal{W}_{1}\left(\mu_{k}(t), \mu(t)\right)=0, \text { for all } t \in[0, T]
$$

Then

$$
\lim _{k} \int_{0}^{\hat{t}}\left\langle\varphi, f_{k}(t, \cdot) \mu_{k}(t)\right\rangle \mathrm{d} t=\int_{0}^{\hat{t}}\langle\varphi, f(t, \cdot) \mu(t)\rangle \mathrm{d} t,
$$

for all $\varphi \in C_{c}^{1}\left(\mathbb{R}^{n}, \mathbb{R}^{d}\right)$ and for all $\hat{t} \in[0, T]$.

Proof. Let us again fix $p>n$. Once we fix $\varphi \in C_{c}^{1}\left(\mathbb{R}^{n}, \mathbb{R}^{d}\right)$, by the assumption $f_{k} \in \mathcal{F}$ we have

$$
\begin{aligned}
\operatorname{Lip}\left(\varphi f_{k}(t, \cdot)\right) & \leq \ell(t)\|\varphi\|_{\infty}+\|\nabla \varphi\|_{\infty}\left\|f_{k}(t, \cdot)\right\|_{L^{\infty}(B(0, R))} \\
& \leq \ell(t)\left(\|\varphi\|_{\infty}+\|\nabla \varphi\|_{\infty}(1+R)\right)
\end{aligned}
$$

where $R>0$ is such that $\operatorname{supp}(\varphi) \Subset B(0, R)$. It follows that

$$
\begin{aligned}
& \underset{k}{\limsup }\left|\int_{0}^{\hat{t}}\left\langle\varphi, f_{k}(t, \cdot) \mu_{k}(t)\right\rangle \mathrm{d} t-\int_{0}^{\hat{t}}\left\langle\varphi, f_{k}(t, \cdot) \mu(t)\right\rangle \mathrm{d} t\right| \\
\leq & \left(\|\varphi\|_{\infty}+\|\nabla \varphi\|_{\infty}(1+R)\right) \underset{k}{\limsup } \int_{0}^{T} \ell(t) \mathcal{W}_{1}\left(\mu_{k}(t), \mu(t)\right) \mathrm{d} t .
\end{aligned}
$$

From (2.11) we have the vanishing pointwise convergence almost everywhere of the latter integrand

$$
\ell(t) \mathcal{W}_{1}\left(\mu_{k}(t), \mu(t)\right) \rightarrow 0, \quad k \rightarrow \infty .
$$

Moreover, by recalling the definition (2.9) and using the uniform first moment condition (2.10), we obtain

$$
\mathcal{W}_{1}\left(\mu_{k}(t), \mu(t)\right) \leq \int_{\mathbb{R}^{n} \times \mathbb{R}^{n}}|x-y| \mathrm{d} \mu_{k}(t, x) \mathrm{d} \mu(t, y) \leq M+\int_{\mathbb{R}^{n}}|y| \mathrm{d} \mu(y),
$$

uniformly with respect to $t \in[0, T]$. Hence, by dominated convergence theorem applied to (2.13) we finally have

$$
\lim _{k \rightarrow \infty}\left|\int_{0}^{\hat{t}}\left\langle\varphi, f_{k}(t, \cdot) \mu_{k}(t)\right\rangle \mathrm{d} t-\int_{0}^{\hat{t}}\left\langle\varphi, f_{k}(t, \cdot) \mu(t)\right\rangle \mathrm{d} t\right|=0 .
$$


Therefore, it is sufficient now to show that

$$
\lim _{k} \int_{0}^{\hat{t}}\left\langle\varphi, f_{k}(t, \cdot) \mu(t)\right\rangle \mathrm{d} t=\int_{0}^{\hat{t}}\langle\varphi, f(t, \cdot) \mu(t)\rangle \mathrm{d} t .
$$

This follows immediately from Corollary 2.7, because $t \rightarrow \varphi \mu(t)$ is a map belonging to the space $L^{\infty}\left([0, \hat{t}], H^{-1, p^{\prime}}\left(\mathbb{R}^{n}, \mathbb{R}^{d}\right)\right)$ with uniform compact support, since $\varphi$ is such.

As in the definition of a weak solution of the equation (1.6) the role of $\varphi$ is played actually by $\nabla_{v} \varphi$ (where $\varphi \in C_{c}^{1}\left(\mathbb{R}^{n}\right)$ ), see formula (4.11) in the proof of Theorem 4.4, we need to extend the validity of Theorem 2.10 as follows.

Corollary 2.11. The statement of Theorem 2.10 actually holds also for $\varphi \in C_{c}^{0}\left(\mathbb{R}^{n}, \mathbb{R}^{d}\right)$.

Proof. Let us notice that

$$
\left|\left\langle\varphi, f_{k}(t, \cdot) \mu_{k}(t)\right\rangle\right| \leq\|\varphi\|_{\infty}\left\|f_{k}(t, \cdot)\right\|_{L^{\infty}(B(0, R))} \leq\|\varphi\|_{\infty} \ell(t)(1+R),
$$

where $R$ is such that $\operatorname{supp}(\varphi) \Subset B(0, R)$. By uniform approximation by functions in $C_{c}^{1}\left(\mathbb{R}^{n}, \mathbb{R}^{d}\right)$, the estimate (2.15) and Theorem 2.10 give the thesis.

The following lower-semicontinuity result will prove to be useful in the proof of Theorem 3.3 and Corollary 4.6. Here the integrability of $\ell$, depending on condition (2.16) below, plays a key role.

Theorem 2.12. Consider a nonnegative convex function $\psi: \mathbb{R}^{d} \rightarrow[0,+\infty)$ satisfying the following condition: there exists a constant $C \geq 0$ and $1 \leq q<+\infty$ such that, for all $R>0$,

$$
\operatorname{Lip}(\psi, B(0, R)) \leq C R^{q-1}
$$

where $B(0, R)$ is the ball of radius $R$ in $\mathbb{R}^{d}$ centered at 0 . For $q$ as in $(2.16)$, fix $\ell \in L^{q}(0, T)$ and consider a sequence of functions $\left(f_{k}\right)_{k \in \mathbb{N}}$ in $\mathcal{F}_{\ell}$ converging to $f$ in the sense of $(2.7)$. Let $\mu_{k}:[0, T] \rightarrow \mathcal{P}_{1}\left(\mathbb{R}^{n}\right)$ be a sequence of functions taking values in the probability measures with finite first moment such that

$$
\operatorname{supp}\left(\mu_{k}(t)\right) \Subset \Omega,
$$

for a.e. $t \in[0, T]$ and $k \in \mathbb{N}$, where $\Omega$ is a relatively compact open set in $\mathbb{R}^{n}$. Let $\mu:[0, T] \rightarrow \mathcal{P}_{1}\left(\mathbb{R}^{n}\right)$, and assume that

$$
\lim _{k} \mathcal{W}_{1}\left(\mu_{k}(t), \mu(t)\right)=0, \text { for a.e. } t \in[0, T] .
$$

Then, we have

$$
\liminf _{k \rightarrow+\infty} \int_{0}^{T}\left\langle\psi\left(f_{k}(t, \cdot)\right), \mu_{k}(t)\right\rangle \mathrm{d} t \geq \int_{0}^{T}\langle\psi(f(t, \cdot)), \mu(t)\rangle \mathrm{d} t .
$$

Remark 2.13. For $\psi$ globally Lipschitz, as in the case of the cost functional (1.5), we can simply take $q=1$.

Proof. We first observe that $(2.17)$ and $(2.18)$ clearly imply that $\operatorname{supp}(\mu(t)) \Subset \Omega$ for a.e. $t \in[0, T]$. Our first goal is to show

$$
\liminf _{k \rightarrow+\infty} \int_{0}^{T}\left\langle\psi\left(f_{k}(t, \cdot)\right), \mu(t)\right\rangle \mathrm{d} t \geq \int_{0}^{T}\langle\psi(f(t, \cdot)), \mu(t)\rangle \mathrm{d} t .
$$

To prove this, we fix $p>n$ and consider $C_{\ell, \Omega}$ as in (2.1).

We define the function $S^{\mu}: L^{q}\left((0, T), W^{1, p}\left(\Omega, \mathbb{R}^{d}\right)\right) \rightarrow[0,+\infty]$ as

$$
S^{\mu}(g):=\left\{\begin{array}{l}
\int_{0}^{T}\langle\psi(g(t, \cdot)), \mu(t)\rangle \mathrm{d} t \quad \text { if } g \in C_{\ell, \Omega} \\
+\infty \text { otherwise. }
\end{array}\right.
$$


We want to prove that $S^{\mu}$ is lower semicontinuous with respect to the weak convergence of $L^{q}((0, T)$, $W^{1, p}\left(\Omega, \mathbb{R}^{d}\right)$ ): with this, $(2.20)$ easily follows. By convexity of $\psi$ and $C_{\ell, \Omega}$, it is immediate to show that $S^{\mu}$ is convex. It then suffices to prove that it is lower semicontinuous in the strong topology of $L^{q}\left((0, T), W^{1, p}\left(\Omega, \mathbb{R}^{d}\right)\right)$ to obtain weak lower semicontinuity. To this end, take a sequence $g_{k} \in L^{q}\left((0, T), W^{1, p}\left(\Omega, \mathbb{R}^{d}\right)\right)$ strongly converging to $g$. The only relevant case is when $g_{k} \in C_{\ell, \Omega}$, so that also $g \in C_{\ell, \Omega}$. In such a case, we clearly have by (2.1) and (2.16) that there exists a constant $C^{\prime}$ only depending on $C$ and the diameter of $\Omega$ such that

$$
\left|\psi\left(g_{k}(t, x, v)\right)-\psi(g(t, x, v))\right| \leq\left(C^{\prime} \ell(t)\right)^{q-1}\left|g_{k}(t, x, v)-g(t, x, v)\right|
$$

for a.e. $t \in[0, T]$ and all $(x, v) \in \Omega$. Denoting with $M$ the continuity constant of Morrey's embedding, we then get

$$
\begin{gathered}
\left|S^{\mu}\left(g_{k}\right)-S^{\mu}(g)\right|=\left|\int_{0}^{T}\left\langle\psi\left(g_{k}(t, \cdot)\right)-\psi(g(t, \cdot)), \mu(t)\right\rangle \mathrm{d} t\right| \\
\leq \int_{0}^{T}\left\|\psi\left(g_{k}(t)\right)-\psi(g(t))\right\|_{L^{\infty}(\Omega)} \mathrm{d} t \leq C^{\prime q-1} \int_{0}^{T} \ell(t)^{q-1}\left\|g_{k}(t)-g(t)\right\|_{L^{\infty}\left(\Omega, \mathbb{R}^{d}\right)} \mathrm{d} t \\
\leq M C^{\prime q-1} \int_{0}^{T} \ell(t)^{q-1}\left\|g_{k}(t)-g(t)\right\|_{W^{1, p}\left(\Omega, \mathbb{R}^{d}\right)} \mathrm{d} t .
\end{gathered}
$$

Now, $\ell(t)^{q-1}$ belongs to $L^{q^{\prime}}(0, T)$ by $q$-integrability of $\ell$, while $\left\|g_{k}(t)-g(t)\right\|_{W^{1, p}\left(\Omega, \mathbb{R}^{d}\right)}$ is going to 0 as $k \rightarrow+\infty$ in $L^{q}(0, T)$. Therefore, Hölder inequality implies

$$
\left|S^{\mu}\left(g_{k}\right)-S^{\mu}(g)\right| \rightarrow 0
$$

thus $(2.20)$ is proved.

We claim now that

$$
\lim _{k \rightarrow+\infty} \int_{0}^{T}\left\langle\psi\left(f_{k}(t, \cdot)\right), \mu_{k}(t)-\mu(t)\right\rangle \mathrm{d} t=0 .
$$

Indeed, since $f_{k} \in \mathcal{F}_{\ell}$ and using (2.16), we have that there exists a constant $C^{\prime}$ only depending on $C$ and on the diameter of $\Omega$ such that

$$
\operatorname{Lip}\left(\psi \circ f_{k}(t), \Omega\right) \leq C^{q-1} \ell(t)^{q}
$$

for all $k \in \mathbb{N}$ and a.e. $t \in(0, T)$, where $\psi \circ f_{k}(t)$ is the composition of the functions $\psi$ and $f_{k}(t)$. Therefore

$$
\begin{aligned}
\left|\int_{0}^{T}\left\langle\psi\left(f_{k}(t, \cdot)\right), \mu_{k}(t)-\mu(t)\right\rangle \mathrm{d} t\right| & =\left|\int_{0}^{T}\left\langle\psi\left(f_{k}(t, \cdot)\right)-\psi\left(f_{k}(t, 0)\right), \mu_{k}(t)-\mu(t)\right\rangle \mathrm{d} t\right| \\
& \leq C^{\prime q-1} \int_{0}^{T} \ell(t)^{q} \mathcal{W}_{1}\left(\mu_{k}(t), \mu(t)\right) \mathrm{d} t .
\end{aligned}
$$

By (2.18), this latter integrand is pointwise vanishing. Since clearly condition (2.17) implies (2.10), by (2.14) $\mathcal{W}_{1}\left(\mu_{k}(t), \mu(t)\right)$ is bounded uniformly with respect to $t$ and $k$; since $\ell \in L^{q}(0, T)$, we can then use the dominated convergence theorem

$$
\int_{0}^{T} \ell(t)^{q} \mathcal{W}_{1}\left(\mu_{k}(t), \mu(t)\right) \mathrm{d} t \rightarrow 0
$$

With this, (2.21) follows: combining it with (2.20), we get (2.19).

As a concluding remark for this section, we point out that, although we consider here controls in the class $\mathcal{F}_{\ell}$ depending on both the variables $x$ and $v$, our analysis apply without any change to controls that depend only on some specific variables. This could be justified by some modeling reasons: for instance, it would be consistent 
with the previous work [11] to take controls which depend exclusively by the velocity state. Indeed, any subclass of $\mathcal{F}$ consisting of controls only depending on some specific variables is closed with respect to the convergence in (2.7), as we clarify in the following remark.

Remark 2.14. For $1 \leq d<n$ we write the generic point $z$ of $\mathbb{R}^{n}$ as $z=(u, w), u \in \mathbb{R}^{n-d}, w \in \mathbb{R}^{d}$. Given $\mathcal{F}_{\ell}$ as in Definition 2.1, we also introduce the following subclass of $\mathcal{F}_{\ell}$ of admissible controls given by

$$
\mathcal{F}_{\ell}^{w}:=\left\{f(t, z) \in \mathcal{F}_{\ell}: f(t, z)=f(t, w)\right\}
$$

Trivially, $f \in \mathcal{F}_{\ell}^{w}$ if and only if $|f(t, 0)|+\left\|\nabla_{w} f(t, \cdot)\right\|_{L^{\infty}\left(\mathbb{R}^{d}\right)} \leq \ell(t)$ for almost every $t \in[0, T]$. We can show that $\mathcal{F}_{\ell}^{w}$ is closed with respect to the weak convergence in Corollary 2.7. Indeed, if $\left(f_{j}\right)_{j \in \mathbb{N}} \in \mathcal{F}_{\ell}^{w}$ is a sequence, then $\left(f_{j}\right)_{j \in \mathbb{N}}$ is also a sequence in $\mathcal{F}_{\ell}$. Assume now that $f_{j}$ is converging to $f \in \mathcal{F}_{\ell}$ in the sense of (2.7). Let $\left\{u_{k}, k \in \mathbb{N}\right\}$ and $\left\{w_{k}, k \in \mathbb{N}\right\}$ be two countable dense subsets of $\mathbb{R}^{n-d}$ and $\mathbb{R}^{d}$, respectively. Pick two different $u_{k_{1}}, u_{k_{2}}$ in the first countable subset, and one fixed $w_{k_{3}}$ in the second one. Fix $t_{1}$ and $t_{2}$ in $[0, T]$ and set

$$
\psi(t):=\chi_{\left(t_{1}, t_{2}\right)}\left(\delta_{u_{k_{1}}}-\delta_{u_{k_{2}}}\right) \otimes \delta_{w_{k_{3}}} .
$$

Since $f_{j} \in \mathcal{F}_{\ell}^{w}$ we have for a.e. $t \in\left(t_{1}, t_{2}\right)$ that

$$
\left\langle f_{j}(t), \psi(t)\right\rangle=f_{j}\left(t, u_{k_{1}}, w_{k_{3}}\right)-f_{j}\left(t, u_{k_{2}}, w_{k_{3}}\right)=f_{j}\left(t, w_{k_{3}}\right)-f_{j}\left(t, w_{k_{3}}\right)=0,
$$

so that (2.7) specifies easily to

$$
0=\int_{t_{1}}^{t_{2}}\left[f\left(t, u_{k_{1}}, w_{k_{3}}\right)-f\left(t, u_{k_{2}}, w_{k_{3}}\right)\right] \mathrm{d} t
$$

By the Lebesgue theorem, we can find a set $N \subset[0, T]$ of zero Lebesgue measure and independent of the elements $u_{k_{1}}, u_{k_{2}}$, and $w_{k_{3}}$ such that

$$
0=f\left(t, u_{k_{1}}, w_{k_{3}}\right)-f\left(t, u_{k_{2}}, w_{k_{3}}\right),
$$

for all $t \in[0, T] \backslash N$. Since $f(t, \cdot, \cdot)$ is continuous for almost every $t$, by a density argument we infer

$$
f\left(t, u_{1}, w\right)-f\left(t, u_{2}, w\right)=0
$$

for all $t \in[0, T] \backslash N, u_{1}$ and $u_{2} \in \mathbb{R}^{n-d}$, and $w$ in $\mathbb{R}^{d}$. This is exactly saying that $f \in \mathcal{F}_{\ell}^{w}$.

\section{The Finite Dimensional CONTROL PROBLEM}

In the following we consider problems in the phase space $\mathbb{R}^{n}$ where $n=2 d$ with state variables $z=(x, v), x$, $v \in \mathbb{R}^{d}$. We state the following assumptions:

(H) Let $H: \mathbb{R}^{2 d} \rightarrow \mathbb{R}^{d}$ be a locally Lipschitz function such that

$$
|H(z)| \leq C(1+|z|), \quad \text { for all } z \in \mathbb{R}^{2 d} .
$$

(L) Let $L: \mathbb{R}^{2 d} \times \mathcal{P}_{1}\left(\mathbb{R}^{2 d}\right) \rightarrow \mathbb{R}_{+}$be a continuous function with respect to the product topology generated by the Euclidean distance on $\mathbb{R}^{2 d}$ and the distance $\mathcal{W}_{1}$ on $\mathcal{P}_{1}\left(\mathbb{R}^{2 d}\right)$.

$(\Psi)$ Let $\psi: \mathbb{R}^{d} \rightarrow[0,+\infty)$ be a nonnegative convex function satisfying the following assumption: there exist $C \geq 0$ and $1 \leq q<+\infty$ such that

$$
\operatorname{Lip}(\psi, B(0, R)) \leq C R^{q-1}
$$

for all $R>0$.

These assumptions are useful in this section, but we shall recall them also in Section 4, where they play again a crucial role. 
Remark 3.1. We discuss a simple sufficient condition for assumption (L) to hold. Consider a continuous kernel $k(x, v, y, w): \mathbb{R}^{4 d} \rightarrow \mathbb{R}^{2 d}$ with the following property: there exist two continuous functions $\alpha(x, v)$ and $\beta(x, v)$ such that

$$
|k(x, v, y, w)| \leq \alpha(x, v)+\beta(x, v)(|y|+|w|) .
$$

Then for all $\mu \in \mathcal{P}_{1}\left(\mathbb{R}^{2 d}\right)$, and all $\alpha>0$ the function

$$
L^{\alpha}(x, v, \mu):=\left|\int_{\mathbb{R}^{2 d}} k(x, v, y, w) \mathrm{d} \mu(y, w)\right|^{\alpha}
$$

are well defined and satisfy $(\mathrm{L})$.

To achieve this, it suffices to check the case $\alpha=1$. Assume indeed that $\left(x_{j}, v_{j}, \mu_{j}\right)$ converges to $(x, v, \mu)$ in the product topology generated by the Euclidean distance on $\mathbb{R}^{2 d}$ and the distance $\mathcal{W}_{1}$ on $\mathcal{P}_{1}\left(\mathbb{R}^{2 d}\right)$. By the properties of the Wasserstein convergence, for fixed $\varepsilon>0$ there exists a compact set $C_{\varepsilon}$ independent of $j$ such that

$$
\int_{\mathbb{R}^{2 d} \backslash C_{\varepsilon}}(1+|y|+|w|) \mathrm{d} \mu_{j} \leq \varepsilon
$$

for all $j$ (see [3], Prop. 7.5.1). By (3.3) we get

$$
\limsup _{j \rightarrow+\infty} \int_{\mathbb{R}^{2 d} \backslash C_{\varepsilon}}\left|k\left(x_{j}, v_{j}, y, w\right)-k(x, v, y, w)\right| \mathrm{d} \mu_{j}(y, w) \leq 2(\alpha(x, v)+\beta(x, v)) \varepsilon .
$$

On the other hand, at fixed $(x, v)$ by the Wasserstein convergence of $\mu_{j}$ to $\mu$ and (3.3) we have

$$
\lim _{j \rightarrow+\infty}\left|\int_{\mathbb{R}^{2 d}} k(x, v, y, w) \mathrm{d} \mu_{j}(y, w)-\int_{\mathbb{R}^{2 d}} k(x, v, y, w) \mathrm{d} \mu(y, w)\right|=0,
$$

while by continuity of $k, k\left(x_{j}, v_{j}, y, w\right)$ converges to $k(x, v, y, w)$ uniformly with respect to $(y, w) \in C_{\varepsilon}$, so that

$$
\lim _{j \rightarrow+\infty} \int_{C_{\varepsilon}}\left|k\left(x_{j}, v_{j}, y, w\right)-k(x, v, y, w)\right| \mathrm{d} \mu_{j}(y, w)=0 .
$$

Combining (3.4) and (3.6) we obtain

$$
\limsup _{j \rightarrow+\infty}\left|\int_{\mathbb{R}^{2 d}} k\left(x_{j}, v_{j}, y, w\right) \mathrm{d} \mu_{j}(y, w)-\int_{\mathbb{R}^{2 d}} k(x, v, y, w) \mathrm{d} \mu(y, w)\right| \leq 2(\alpha(x, v)+\beta(x, v)) \varepsilon,
$$

which proves (L) by arbitrariness of $\varepsilon$.

For $\alpha=2$ and $k(x, v, y, w)=(0, v-w)$, with 0 being the null vector of $\mathbb{R}^{d}$, we get for instance that the cost function associated to the Cucker-Smale system (1.4) and (1.5)

$$
(x, v, \mu) \rightarrow\left|v-\int_{\mathbb{R}^{2 d}} w \mathrm{~d} \mu(y, w)\right|^{2}
$$

actually satisfies (L).

We fix $q$ so that (3.2) holds, and a function $\ell \in L^{q}(0, T)$. Given $N \in \mathbb{N}$ and an initial datum $\left(x_{1}(0), \ldots, x_{N}(0), v_{1}(0), \ldots, v_{N}(0)\right) \in\left(\mathbb{R}^{d}\right)^{N} \times\left(\mathbb{R}^{d}\right)^{N}$, we consider the following optimal control problem:

$$
\min _{f \in \mathcal{F}_{\ell}} \int_{0}^{T} \int_{\mathbb{R}^{2 d}}\left[L\left(x, v, \mu_{N}(t)\right)+\psi(f(t, x, v)] \mathrm{d} \mu_{N}(t)(x, v) \mathrm{d} t,\right.
$$


where

$$
\mu_{N}(t)(x, v)=\frac{1}{N} \sum_{j=1}^{N} \delta_{\left(x_{i}(t), v_{i}(t)\right)}(x, v),
$$

is the time dependent empirical atomic measure supported on the phase space trajectories $\left(x_{i}(t), v_{i}(t)\right) \in \mathbb{R}^{2 d}$, for $i=1, \ldots N$, constrained by being the solution of the system

$$
\left\{\begin{array}{l}
\dot{x}_{i}=v_{i} \\
\dot{v}_{i}=\left(H \star \mu_{N}\right)\left(x_{i}, v_{i}\right)+f\left(t, x_{i}, v_{i}\right), i=1, \ldots N, \quad t \in[0, T]
\end{array}\right.
$$

Let us stress that the existence of Carathéodory solutions of (3.9) for any given $f \in \mathcal{F}_{\ell}$ is actually ensured by Theorems A.1 and A.2 recalled in the Appendix. In equation (3.7), as well as in what follows, the symbol $\mathrm{d} \mu(t)(x, v)$ denotes the integration with respect to a time-dependent probability measure $\mu(t)$ in the variables $(x, v)$. We start with a trajectory confinement result.

Lemma 3.2. Let $f \in \mathcal{F}_{\ell}$ and $(x(t), v(t))$ be the solution of (3.9) with initial datum $(x(0), v(0))$. Then

$$
\mathcal{V}(t) \leq\left\{\mathcal{V}(0)+[1+\mathcal{X}(0)]\left(2 C T+\int_{0}^{t} \ell(s) \mathrm{d} s\right)\right\} \mathrm{e}^{(1+T) \int_{0}^{t}[2 C+\ell(s)] \mathrm{d} s},
$$

for all $t \in[0, T]$, where $\mathcal{V}(t)=\max _{i=1, \ldots, N}\left|v_{i}(t)\right|$ and $\mathcal{X}(t)=\max _{i=1, \ldots, N}\left|x_{i}(t)\right|$. Hence the trajectory $(x(t), v(t))$ is uniformly bounded with respect to $t \in[0, T]$, independently of the number $N$ of particles.

Proof. By integration of (3.9) and the linear growths of both $H$ and control function $f \in \mathcal{F}_{\ell}$, we obtain

$$
\mathcal{V}(t) \leq \mathcal{V}(0)+\int_{0}^{t}[2 C(1+\mathcal{X}(s)+\mathcal{V}(s))+\ell(s)(1+\mathcal{X}(s)+\mathcal{V}(s))] \mathrm{d} s
$$

Moreover, for any $0 \leq s \leq t$

$$
\left|x_{i}(s)\right| \leq\left|x_{i}(0)\right|+\int_{0}^{s}\left|v_{i}(r)\right| \mathrm{d} r \leq\left|x_{i}(0)\right|+\int_{0}^{t}\left|v_{i}(r)\right| \mathrm{d} r
$$

which combined with (3.11) gives

$$
\mathcal{V}(t) \leq\left\{\mathcal{V}(0)+[1+\mathcal{X}(0)]\left(2 C T+\int_{0}^{t} \ell(s) \mathrm{d} s\right)\right\}+(1+T) \int_{0}^{t}[2 C+\ell(s)] \mathcal{V}(s) \mathrm{d} s .
$$

Gronwall's lemma eventually yields (3.10).

From the uniform support bound on $\mu_{N}$ provided by Lemma 3.2 and from Theorems 2.10 and 2.12 we deduce the following well-posedness result.

Theorem 3.3. The finite horizon optimal control problem (3.7) and (3.9) with initial datum $(x(0), v(0))$ has solutions.

Proof. Let us consider a minimizing sequence $\left(f_{j}\right)_{j \in \mathbb{N}}$ in $\mathcal{F}_{\ell}$ and its subsequence $\left(f_{j_{k}}\right)_{k \in \mathbb{N}}$ and $f \in \mathcal{F}_{\ell}$ as in Corollary 2.7. For simplicity we rename $f_{k}=f_{j_{k}}$. Let us also fix $\left(x^{k}(t), v^{k}(t)\right)$ the trajectory solutions to (3.9) corresponding to $f_{k}$.

As we have from (3.9) that

$$
\max _{i=1, \ldots, N}\left|\dot{v}_{i}^{k}(t)\right| \leq 2 C\left(1+\max _{i=1, \ldots, N}\left|x_{i}^{k}(t)\right|+\max _{i=1, \ldots, N}\left|v_{i}^{k}(t)\right|\right)+\ell(t)\left(1+\max _{i=1 \ldots N}\left|v_{i}^{k}(t)\right|\right),
$$


Lemma 3.2 implies the equi-integrability of $\dot{v}_{i}^{k}(t)$, the equi-boundedness and the equi-absolute continuity of $v_{i}^{k}(t)$, hence the equi-Lispchitzianity of $x_{i}^{k}(t)$ as well, uniformly with respect to $k$, for all $i=1, \ldots, N$. By Ascoli-Arzelà theorem there exist a subsequence, again renamed $\left(x^{k}(t), v^{k}(t)\right)_{k \in \mathbb{N}}$ and an absolutely continuous trajectory $(x(t), v(t))$ in $[0, T]$ such that, for $k \rightarrow \infty$

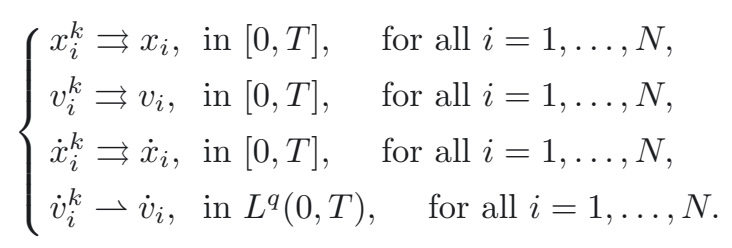

Let us remark that the $\dot{v}_{i}^{k} \rightarrow \dot{v}_{i}$ in $L^{q}(0, T)$ can be seen again as a consequence of the more general Theorem 2.5. In particular $\dot{x}_{i}(t)=v_{i}(t)$ in $[0, T]$, for all $i=1, \ldots, N$. Let us denote

$$
\mu_{N}^{k}(t)=\frac{1}{N} \sum_{i=1}^{N} \delta_{\left(x_{i}^{k}(t), v_{i}^{k}(t)\right)} \quad \text { and } \mu_{N}(t)=\frac{1}{N} \sum_{i=1}^{N} \delta_{\left(x_{i}(t), v_{i}(t)\right)} .
$$

As a consequence of Lemma 3.2 and of the uniform convergence of the trajectories we have that for every $i=1, \ldots, N$

$$
\mathcal{W}_{1}\left(\delta_{\left(x_{i}^{k}(t), v_{i}^{k}(t)\right)}, \delta_{\left(x_{i}(t), v_{i}(t)\right)}\right) \rightarrow 0 \quad \text { and } \quad \mathcal{W}_{1}\left(\mu_{N}^{k}(t), \mu_{N}(t)\right) \rightarrow 0
$$

as $k \rightarrow+\infty$, uniformly in $t \in[0, T]$. Furthermore there exists a relatively compact open subset $\Omega \subset \mathbb{R}^{2 d}$ such that

$$
\operatorname{supp}\left(\mu_{N}^{k}(t)\right) \cup \operatorname{supp}\left(\mu_{N}(t)\right) \Subset \Omega,
$$

for all $t \in[0, T]$ and $k \in \mathbb{N}$. By the linear growth of $H$ we deduce

$$
H \star \mu_{N}^{k}\left(x_{i}^{k}, v_{i}^{k}\right) \rightrightarrows H \star \mu_{N}\left(x_{i}, v_{i}\right), \text { in }[0, T], \quad \text { for } k \rightarrow \infty,
$$

see also Lemma A.7 in the Appendix.

To prove that $(x(t), v(t))$ is actually the Carathéodory solution of (3.9) associated to the forcing term $f$, we therefore have only to show that for all $i=1, \ldots, N$ one has

$$
\dot{v}_{i}=\left(H \star \mu_{N}\right)\left(x_{i}, v_{i}\right)+f\left(t, x_{i}, v_{i}\right) .
$$

This is clearly equivalent to the following: for every $\xi \in \mathbb{R}^{d}$ and every $\hat{t} \in[0, T]$ it holds:

$$
\xi \cdot \int_{0}^{\hat{t}} \dot{v}_{i}(t) \mathrm{d} t=\xi \cdot \int_{0}^{\hat{t}}\left[\left(H \star \mu_{N}(t)\right)\left(x_{i}(t), v_{i}(t)\right)+f\left(t, x_{i}(t), v_{i}(t)\right)\right] \mathrm{d} t .
$$

In view of the weak $L^{q}$ convergence of $\dot{v}_{i}^{k}$ to $\dot{v}_{i}$ and of (3.15), (3.17) actually reduces to show

$$
\lim _{k \rightarrow+\infty} \xi \cdot \int_{0}^{\hat{t}} f\left(t, x_{i}^{k}(t), v_{i}^{k}(t)\right) \mathrm{d} t=\xi \cdot \int_{0}^{\hat{t}} f\left(t, x_{i}(t), v_{i}(t)\right) \mathrm{d} t .
$$

Given $\varphi_{\xi} \in C_{c}^{1}\left(\mathbb{R}^{2 d}, \mathbb{R}^{d}\right)$ such that $\varphi_{\xi} \equiv \xi$ in $\Omega$, the above equality reduces to

$$
\lim _{k \rightarrow+\infty} \int_{0}^{\hat{t}}\left\langle\varphi_{\xi}, f(t, \cdot) \delta_{\left(x_{i}^{k}(t), v_{i}^{k}(t)\right)}\right\rangle \mathrm{d} t=\int_{0}^{\hat{t}}\left\langle\varphi_{\xi}, f(t, \cdot) \delta_{\left(x_{i}(t), v_{i}(t)\right)}\right\rangle \mathrm{d} t
$$

which follows from (2.12), (3.13), and (3.14). 
Using again (3.13) and (3.14), the inequality

$$
\liminf _{k \rightarrow+\infty} \int_{0}^{T} \int_{\mathbb{R}^{2 d}} \psi\left(f_{k}(t, x, v)\right) \mathrm{d} \mu_{N}^{k}(t)(x, v) \mathrm{d} t \geq \int_{0}^{T} \int_{\mathbb{R}^{2 d}} \psi(f(t, x, v)) \mathrm{d} \mu_{N}(t)(x, v) \mathrm{d} t
$$

follows now directly from (2.19). Furthermore, condition (L) and the uniform convergences in (3.12) and (3.15) yield that $L\left(x_{i}^{k}(t), v_{i}^{k}(t), \mu_{N}^{k}(t)\right) \rightrightarrows L\left(x_{i}(t), v_{i}(t), \mu_{N}(t)\right)$ uniformly in $[0, T]$ as $k \rightarrow+\infty$ for all $i=1, \ldots, N$, so that

$$
\begin{aligned}
\lim _{k} \int_{0}^{T} \int_{\mathbb{R}^{2 d}} L\left(x, v, \mu_{N}^{k}(t)\right) \mathrm{d} \mu_{N}^{k}(t)(x, v) \mathrm{d} t & =\lim _{k} \int_{0}^{T} \frac{1}{N} \sum_{i=1}^{N} L\left(x_{i}^{k}(t), v_{i}^{k}(t), \mu_{N}^{k}(t)\right) \mathrm{d} t \\
& =\int_{0}^{T} \frac{1}{N} \sum_{i=1}^{N} L\left(x_{i}(t), v_{i}(t), \mu_{N}(t)\right) \mathrm{d} t \\
& =\int_{0}^{T} \int_{\mathbb{R}^{2 d}} L\left(x, v, \mu_{N}(t)\right) \mathrm{d} \mu_{N}(t)(x, v) \mathrm{d} t .
\end{aligned}
$$

Combining (3.18) and (3.19) gives the optimality of $f$ as a minimizer of (3.7) under the solution constraint (3.9).

\section{MeAN-FiELD SOLUtions}

In this section we are concerned with the limit for $N \rightarrow \infty$ of the solutions of the ODE system (1.1) to solutions of the PDE of the type (1.6). First we need to define a proper concept of solution for (1.6). To this aim, assuming the $L^{1}$ integrability of the function $\ell$ defining the class $\mathcal{F}_{\ell}$ is sufficient.

Definition 4.1. Let $\ell \in L^{1}(0, T)$. Fix a function $f$ belonging to the class $\mathcal{F}_{\ell}$. Given a locally Lipschitz function $H: \mathbb{R}^{2 d} \rightarrow \mathbb{R}^{d}$ satisfying (3.1), we say that a map $\mu:[0, T] \rightarrow \mathcal{P}_{1}\left(\mathbb{R}^{2 d}\right)$ continuous with respect to $\mathcal{W}_{1}$ is a weak equi-compactly supported solution of the equation

$$
\frac{\partial \mu}{\partial t}+v \cdot \nabla_{x} \mu=\nabla_{v} \cdot[(H \star \mu+f) \mu]
$$

with forcing term $f$ on the interval $[0, T]$ if there exists $R>0$ such that

$$
\operatorname{supp} \mu(t) \subset B(0, R)
$$

for every $t \in[0, T]$, and, defining $w_{H, \mu, f}(t, x, v):[0, T] \times \mathbb{R}^{d} \times \mathbb{R}^{d} \rightarrow \mathbb{R}^{d} \times \mathbb{R}^{d}$ as

$$
w_{H, \mu, f}(t, x, v):=(v, H \star \mu(t)(x, v)+f(t, x, v))
$$

one has

$$
\frac{\mathrm{d}}{\mathrm{d} t} \int_{\mathbb{R}^{2 d}} \zeta(x, v) \mathrm{d} \mu(t)(x, v)=\int_{\mathbb{R}^{2 d}} \nabla \zeta(x, v) \cdot w_{H, \mu, f}(t, x, v) \mathrm{d} \mu(t)(x, v)
$$

for every $\zeta \in C_{c}^{\infty}\left(\mathbb{R}^{d} \times \mathbb{R}^{d}\right)$, in the sense of distributions.

Since the linear span of functions of the type $\eta(t) \zeta(x, v)$, with $\eta \in C_{c}^{\infty}(0, T)$ and $\zeta \in C_{c}^{\infty}\left(\mathbb{R}^{d} \times \mathbb{R}^{d}\right)$ is dense in $C_{c}^{1}\left((0, T) \times \mathbb{R}^{d} \times \mathbb{R}^{d}\right)$ arguing as in [3], Remark 8.1.1 one can see that (4.3) is equivalent to saying that

$$
\int_{0}^{T} \int_{\mathbb{R}^{2 d}}\left(\partial_{t} \varphi(t, x, v)+\nabla_{x, v} \varphi(t, x, v) \cdot w_{H, \mu, f}(t, x, v)\right) \mathrm{d} \mu(t)(x, v) \mathrm{d} t=0
$$


for every $\varphi \in C_{c}^{1}\left((0, T) \times \mathbb{R}^{d} \times \mathbb{R}^{d}\right)$. Using also the explicit expression of $w_{H, \mu, f}$, integrating between 0 and $\hat{t} \in(0, T]$ we can equivalently reformulate (4.3) by asking that the equality

$$
\begin{gathered}
\int_{\mathbb{R}^{2 d}} \zeta(x, v) \mathrm{d} \mu(\hat{t})(x, v)-\int_{\mathbb{R}^{2 d}} \zeta(x, v) \mathrm{d} \mu(0)(x, v) \\
=\int_{0}^{\hat{t}} \int_{\mathbb{R}^{2 d}}\left(\nabla_{x} \zeta(x, v) \cdot v+\nabla_{v} \zeta(x, v) \cdot H \star \mu(t)(x, v)+\nabla_{v} \zeta(x, v) \cdot f(t, x, v)\right) \mathrm{d} \mu(t)(x, v)
\end{gathered}
$$

holds for every $\hat{t} \in[0, T]$ and every $\zeta \in C_{c}^{\infty}\left(\mathbb{R}^{d} \times \mathbb{R}^{d}\right)$. Finally, we can also consider test functions depending also on $t$, and defining solutions as satisfying the equality

$$
0=\int_{0}^{T} \int_{\mathbb{R}^{2 d}}\left(\partial_{t} \varphi(t, x, v)+\nabla_{x} \varphi(t, x, v) \cdot v+\nabla_{v} \varphi(t, x, v) \cdot H \star \mu(t)(x, v)+\nabla_{v} \varphi(t, x, v) \cdot f(t, x, v)\right) \mathrm{d} \mu(t)(x, v),
$$

for every $\varphi \in C_{c}^{1}\left((0, T) \times \mathbb{R}^{d} \times \mathbb{R}^{d}\right)$ : again, this is equivalent to (4.3).

Remark 4.2. Observe that $w_{H, \mu, f}(t, x, v)$ is a Carathéodory vector field, thus measurable with respect to any product measure of the type $\mathcal{L}^{1} \times \mu$ with $\mathcal{L}^{1}$ the Lebesgue measure on $[0, T]$ and $\mu$ a Borel probability measure on $\mathbb{R}^{d} \times \mathbb{R}^{d}$. Furthermore, by (3.1) and (4.2), we can show that (A.7) in the Appendix holds for $n=2 d$ and $p=d$. Taking also into account (A.9) of Lemma A.4 for $n=2 d$ and $p=d$ and (A.11) in the Appendix, it follows that the $W^{1, \infty}$ norm of $w_{H, \mu, f}(t, \cdot, \cdot)$ is bounded by an $L^{1}$-function of $t$ on any compact subset of $\mathbb{R}^{d} \times \mathbb{R}^{d}$. Jointly with the aforementioned measurability property, this allows us to repeat the arguments in ([3], Sect. 8.1) proving that $\mu(t)$ is a weak equi-compactly supported solution of (4.1) with forcing term $f$ on the interval $[0, T]$ if and only if it satisfies (4.2) and the measure-theoretical fixed point equation

$$
\mu(t)=\left(\mathcal{T}_{t}^{\mu}\right)_{\sharp} \mu_{0},
$$

with $\mu_{0}:=\mu(0)$ and $\mathcal{T}_{t}^{\mu}$ is the flow function defined by (A.12) in the Appendix. Here $\left(\mathcal{T}_{t}^{\mu}\right)_{\sharp}$ denotes the push-forward of $\mu_{0}$ through $\mathcal{T}_{t}^{\mu}$.

\subsection{Empirical equi-compactly supported solutions}

Let us now again fix $\ell \in L^{1}(0, T)$ and a locally Lipschitz function $H: \mathbb{R}^{2 d} \rightarrow \mathbb{R}^{d}$ satisfying (3.1). Given $N \in \mathbb{N}$, an initial datum $\left(x_{1}^{0}, \ldots, x_{N}^{0}, v_{1}^{0}, \ldots, v_{N}^{0}\right) \in B\left(0, R_{0}\right) \subset\left(\mathbb{R}^{d}\right)^{N} \times\left(\mathbb{R}^{d}\right)^{N}$, with $R_{0}>0$ independent of $N$, and a function $f_{N} \in \mathcal{F}_{\ell}$, perhaps also depending on $N$, we consider the following time dependent empirical atomic measure

$$
\mu_{N}(t)(x, v)=\frac{1}{N} \sum_{j=1}^{N} \delta_{\left(x_{i}(t), v_{i}(t)\right)}(x, v),
$$

supported on the phase space trajectories $\left(x_{i}(t), v_{i}(t)\right) \in \mathbb{R}^{2 d}$, for $i=1, \ldots N$, defining the solution of the system

$$
\left\{\begin{array}{l}
\dot{x}_{i}=v_{i} \\
\dot{v}_{i}=\left(H \star \mu_{N}\right)\left(x_{i}, v_{i}\right)+f_{N}\left(t, x_{i}, v_{i}\right), i=1, \ldots, N, \quad t \in[0, T]
\end{array}\right.
$$

with initial datum $(x(0), v(0))=\left(x^{0}, v^{0}\right)$. The symbol $\star$ indicates the convolution operator of a function with respect to a measure. We remark again that under our assumptions on $f$ and $H$, existence and uniqueness of Carathéodory solutions of (4.8) are ensured (see Appendix for details) hence the well-posedness of (4.7).

Lemma 4.3. Under the assumptions considered since the beginning of this section, let us define $\mu_{N}$ as in (4.7). The the following properties hold:

(a) $\operatorname{supp}\left(\mu_{N}(t)\right) \subset B\left(0, R_{T}\right)$, where $R_{T}>0$ is independent of $N$; 
(b) $\mathcal{W}_{1}\left(\mu_{N}\left(t_{1}\right), \mu_{N}\left(t_{2}\right)\right) \leq L_{T} \int_{t_{1}}^{t_{2}}(1+\ell(s)) d s$, for all $t_{1}, t_{2} \in[0, T]$, for a suitable constant $L_{T}>0$ dependent on $T$ and independent of $N$;

(c) for all $\hat{t} \in[0, T]$ and for all $\zeta \in C_{c}^{1}\left(\mathbb{R}^{2 d}\right)$

$$
\left\langle\zeta, \mu_{N}(\hat{t})-\mu_{N}(0)\right\rangle=\int_{0}^{\hat{t}}\left[\int_{\mathbb{R}^{2 d}} \nabla \zeta(x, v) \cdot w_{H, \mu_{N}, f_{N}}(t, x, v) \mathrm{d} \mu_{N}(t, x, v)\right] \mathrm{d} t .
$$

In particular the maps $t \rightarrow \mu_{N}(t)$ are equi-compactly supported solutions of the equation (4.1) in the sense of Definition 4.1 for all $N \in \mathbb{N}$.

Proof. The property (a) is a direct consequence of the equi-boundedness of the datum $\left(x_{1}(0), \ldots, x_{N}(0)\right.$, $\left.v_{1}(0), \ldots, v_{N}(0)\right) \in B\left(0, R_{0}\right)$, and of Lemma 3.2. Let us prove (b). As the measures $\mu_{N}\left(t_{1}\right)$ and $\mu_{N}\left(t_{2}\right)$ are actually atomic, in this case the 1-Wasserstein distance can be estimated in terms of the $\ell^{1}$-norm of the Euclidean distances of the respective supporting atoms:

$$
\mathcal{W}_{1}\left(\mu_{N}\left(t_{1}\right), \mu_{N}\left(t_{2}\right)\right) \leq \frac{1}{N} \sum_{i=1}^{N}\left(\left|x_{i}\left(t_{1}\right)-x_{i}\left(t_{2}\right)\right|+\left|v_{i}\left(t_{1}\right)-v_{i}\left(t_{2}\right)\right|\right) .
$$

As $\dot{x}_{i}(t)=v_{i}(t)$, from (a) we know already that $\left|x_{i}\left(t_{1}\right)-x_{i}\left(t_{2}\right)\right| \leq L_{T}\left|t_{1}-t_{2}\right|$ for all $i=1, \ldots N$, for a suitable constant $L_{T}>0$. In the following $L_{T}$ may be a different constant at different places, but always dependent on $T$, and independent of $N$. As we have

$$
\max _{i=1, \ldots, N}\left|\dot{v}_{i}(t)\right| \leq 2 C\left(1+\max _{i=1, \ldots, N}\left|x_{i}(t)\right|+\max _{i=1, \ldots, N}\left|v_{i}(t)\right|\right)+\ell(t)\left(1+\max _{i=1, \ldots, N}\left|x_{i}(t)\right|+\max _{i=1 \ldots N}\left|v_{i}(t)\right|\right),
$$

Lemma 3.2 implies the equi-integrability of $\dot{v}_{i}(t)$, and also $\left|v_{i}\left(t_{1}\right)-v_{i}\left(t_{2}\right)\right| \leq L_{T} \int_{t_{1}}^{t_{2}}(1+\ell(s)) d s$ for all $i=1, \ldots N$, for a suitable $L_{T}$ independent of $N$. With this, (b) follows. The validity of (c) is a standard argument, which is developed by considering the differentiation

$$
\begin{aligned}
\frac{\mathrm{d}}{\mathrm{d} t}\left\langle\zeta, \mu_{N}(t)\right\rangle & =\frac{1}{N} \frac{\mathrm{d}}{\mathrm{d} t} \sum_{i=1}^{N} \zeta\left(x_{i}(t), v_{i}(t)\right) \\
& =\frac{1}{N}\left[\sum_{i=1}^{N} \nabla_{x} \zeta\left(x_{i}(t), v_{i}(t)\right) \cdot \dot{x}_{i}(t)+\sum_{i=1}^{N} \nabla_{v} \zeta\left(x_{i}(t), v_{i}(t)\right) \cdot \dot{v}_{i}(t)\right],
\end{aligned}
$$

and directly applying the substitutions as in (4.8).

\subsection{Convergence of empirical solutions}

In this section we show how solutions to (4.8) converges to solutions of (4.1) in the sense of Definition 4.1.

Theorem 4.4. Let us consider a sequence of equi-compactly supported empirical probability measures $\left(\mu_{N}^{0}\right)_{N \in \mathbb{N}}$, where $\mu_{N}^{0}=\frac{1}{N} \sum_{i=1}^{M} \delta_{\left(\left(x_{N}^{0}\right)_{i},\left(v_{N}^{0}\right)_{i}\right)}(x, v)$ for suitable sets of points $\left(x_{N}^{0}, v_{N}^{0}\right)$ in $\left(\mathbb{R}^{d}\right)^{N} \times\left(\mathbb{R}^{d}\right)^{N}$ with the properties

(i) $\mu_{N}^{0}$ has support equi-bounded in $\mathbb{R}^{2 d}$, i.e., $\left(x_{N}^{0}, v_{N}^{0}\right) \in B\left(0, R_{0}\right)$, for $R_{0}>0$ independent of $N$;

(ii) there exists a compactly supported $\mu^{0} \in \mathcal{P}_{1}\left(\mathbb{R}^{2 d}\right)$ such that $\lim _{N \rightarrow \infty} \mathcal{W}_{1}\left(\mu_{N}^{0}, \mu^{0}\right)=0$.

Given $\ell \in L^{1}(0, T)$ and $\left(f_{N}\right)_{N \in \mathbb{N}}$ an arbitrary sequence in $\mathcal{F}_{\ell}$, we can accordingly define $\mu_{N}(t)$ as the empirical equi-compactly supported solutions (4.7) of (4.8) with initial data $\left(x_{N}^{0}, v_{N}^{0}\right)$ in $\left(\mathbb{R}^{d}\right)^{N} \times\left(\mathbb{R}^{d}\right)^{N}$ and forcing term $f_{N}$, respectively, for all $N \in \mathbb{N}$.

Then there exist a subsequence $\left(f_{N_{k}}\right)_{k \in \mathbb{N}}$ converging in the sense of $(2.7)$ to a function $f \in \mathcal{F}_{\ell}$ such that the corresponding subsequence $\left(\mu_{N_{k}}(t)\right)_{k \in \mathbb{N}}$ converges in Wasserstein distance uniformly in $t \in[0, T]$ to a weak equi-compactly supported solution $\mu(t)$ of equation (4.1) with forcing term $f$ and initial condition $\mu(0)=\mu^{0}$ in the sense of Definition 4.1. 
Proof. Thanks to the equi-boundedness assumption (i) we can apply Lemma 4.3(a) and (b), and the sequence of measures $\left(\mu_{N}(t)\right)_{N \in \mathbb{N}}$ is actually equi-bounded and equi-continuous. By an application of the Ascoli-Arzelà theorem for functions on $[0, T]$ with values in the complete metric space $\left(\mathcal{P}_{1}\left(B\left(0, R_{T}\right)\right), \mathcal{W}_{1}\right)$ (here $\mathcal{P}_{1}\left(B\left(0, R_{T}\right)\right)$ actually denotes the space of probability measures compactly supported in $B\left(0, R_{T}\right)$, endowed with the 1-Wasserstein distance), up to extracting a subsequence, we deduce the existence of a uniform limit $\mu(t)$ in 1-Wasserstein distance, which is actually equi-supported in $B\left(0, R_{T}\right)$, uniformly with respect to $t \in[0, T]$, for $R_{T}>0$ as in Lemma 4.3(a). Obviously it is also $\mu(0)=\mu^{0}$, and by lower-semicontinuity of the Wasserstein distance with respect to the narrow convergence we have

$$
\mathcal{W}_{1}\left(\mu\left(t_{2}\right), \mu\left(t_{1}\right)\right) \leq L_{T} \int_{t_{1}}^{t_{2}}(1+\ell(s)) \mathrm{d} s,
$$

for all $t_{1}, t_{2} \in[0, T]$, where $L_{T}>0$ is as in Lemma $4.3(\mathrm{~b})$. Moreover,

$$
\lim _{k \rightarrow \infty}\left\langle\zeta, \mu_{N_{k}}(\hat{t})-\mu_{N_{k}}(0)\right\rangle=\langle\zeta, \mu(\hat{t})-\mu(0)\rangle,
$$

for all $\zeta \in C_{c}^{1}\left(\mathbb{R}^{2 d}\right)$ and $\hat{t} \in[0, T]$. By possibly extracting an additional subsequence, which we do not relabel, thanks to Corollary 2.7 we can assume that there exists $f \in \mathcal{F}_{\ell}$ so that $\left(f_{N_{k}}\right)_{k \in \mathbb{N}} \rightarrow f$ in the sense of (2.7), and by applying Theorem 2.10 and Corollary 2.11 we immediately obtain for any $\hat{t} \in[0, T]$

$$
\lim _{k \rightarrow \infty} \int_{0}^{\hat{t}} \int_{\mathbb{R}^{2 d}}\left(\nabla_{v} \zeta(x, v) \cdot f_{N_{k}}(t, x, v)\right) \mathrm{d} \mu_{N_{k}}(t)(x, v) \mathrm{d} t=\int_{0}^{\hat{t}} \int_{\mathbb{R}^{2 d}}\left(\nabla_{v} \zeta(x, v) \cdot f(t, x, v)\right) \mathrm{d} \mu(t)(x, v) \mathrm{d} t,
$$

and by weak-* convergence and the dominated convergence theorem

$$
\lim _{k \rightarrow \infty} \int_{0}^{\hat{t}} \int_{\mathbb{R}^{2 d}}\left(\nabla_{v} \zeta(x, v) \cdot v\right) \mathrm{d} \mu_{N_{k}}(t)(x, v) \mathrm{d} t=\int_{0}^{\hat{t}} \int_{\mathbb{R}^{2 d}}\left(\nabla_{v} \zeta(x, v) \cdot v\right) \mathrm{d} \mu(t)(x, v) \mathrm{d} t,
$$

for all $\zeta \in C_{c}^{1}\left(\mathbb{R}^{2 d}\right)$. By Lemma A.7 in Appendix we also obtain that for every $\rho>0$

$$
\lim _{k \rightarrow \infty}\left\|H \star \mu_{N_{k}}(t)-H \star \mu(t)\right\|_{L^{\infty}(B(0, \rho))}=0,
$$

uniformly in $t \in[0, T]$ and, as $\zeta \in C_{c}^{1}\left(\mathbb{R}^{2 d}\right)$ has compact support, it follows that

$$
\lim _{k \rightarrow \infty}\left\|\nabla_{v} \zeta \cdot\left(H \star \mu_{N_{k}}(t)-H \star \mu(t)\right)\right\|_{\infty}=0 .
$$

Since the product measures $\mathcal{L}^{1}\left\llcorner_{[0, \hat{t}]} \times \frac{1}{\hat{t}} \mu_{N_{k}}(t)\right.$ converge in $\mathcal{P}_{1}\left([0, \hat{t}] \times \mathbb{R}^{2 d}\right)$ to $\mathcal{L}^{1}\left\llcorner_{[0, \hat{t}]} \times \frac{1}{\hat{t}} \mu(t)\right.$, we obtain also

$$
\lim _{k \rightarrow \infty} \int_{0}^{\hat{t}} \int_{\mathbb{R}^{2 d}}\left(\nabla_{v} \zeta(x, v) \cdot H \star \mu_{N_{k}}(t)\right) \mathrm{d} \mu_{N_{k}}(t)(x, v) \mathrm{d} t=\int_{0}^{\hat{t}} \int_{\mathbb{R}^{2 d}}\left(\nabla_{v} \zeta(x, v) \cdot H \star \mu(t)\right) \mathrm{d} \mu(t)(x, v) \mathrm{d} t,
$$

The statement now follows by combining (4.9) and (4.13).

Remark 4.5. The rigorous derivation of the mean-field limit of equi-compactly supported solutions (4.7) of (4.8) to weak-solutions of (4.1) in the sense of Definition 4.1 in situations where no control is addressed, i.e., when $f_{N} \equiv 0$ for all $N \in \mathbb{N}$, has been already considered, for instance, in [10] and we refer also to the very recent survey paper [12]. Nevertheless, although it may be seen as a minor extension, the situation where a sequence of nontrivial discontinuous-in-time controls $\left(f_{N}\right)_{N \in \mathbb{N}}$ is present in the equations requires to generalize the results in [10] to solutions of Carathéodory for (4.8) [32]. As already metioned we sketched these generalizations in the Appendix for the sake of completeness. In particular, as a consequence of Theorem 4.4, existence, uniqueness, and stability of weak measure-valued solutions to (4.1) with compactly supported data will be 
given in details below in Theorems 4.7 and A.8. The difficulty to be considered is that, due to the potential discontinuous nature in time of the functions, the sequence $\left(f_{N}\right)_{N \in \mathbb{N}}$ possesses limits exclusively in the very weak topology defined by (2.7). In particular any subsequence $f_{N_{k}}(t)$ does not in general weakly converge to a function $f(t) \in W_{\text {loc }}^{1, \infty}\left(\mathbb{R}^{2 d}, \mathbb{R}^{d}\right)$ pointwise in time, as we already stated in Remark 2.9. From this subtle difficulty it comes the necessity of developing Theorem 2.10 and Corollary 2.11 as tools for the proof of Theorem 4.4.

We now prove the lower semicontinuity of the cost functionals with respect to the convergences in the previous theorem, what can be seen as a $\Gamma-\lim$ inf condition.

Corollary 4.6. Let us now assume as in $(L)$ that $L: \mathbb{R}^{2 d} \times \mathcal{P}_{1}\left(\mathbb{R}^{2 d}\right) \rightarrow \mathbb{R}_{+}$is a continuous function with respect to the product topology generated by the Euclidean distance on $\mathbb{R}^{2 d}$ and the distance $\mathcal{W}_{1}$ on $\mathcal{P}_{1}\left(\mathbb{R}^{2 d}\right)$. Consider a nonnegative convex function $\psi: \mathbb{R}^{d} \rightarrow[0,+\infty)$ satisfying condition $(\Psi)$. Besides the assumptions of Theorem 4.4, suppose that $\ell \in L^{q}(0, T)$, with $1 \leq q<+\infty$ being as in (3.2). We then have the following lower-semicontinuity property

$$
\begin{aligned}
\liminf _{k \rightarrow \infty} & \int_{0}^{T} \int_{\mathbb{R}^{2 d}}\left(L\left(x, v, \mu_{N_{k}}(t)\right)+\psi\left(f_{N_{k}}(t, x, v)\right)\right) \mathrm{d} \mu_{N_{k}}(t)(x, v) \\
\geq & \int_{0}^{T} \int_{\mathbb{R}^{2 d}}(L(x, v, \mu(t))+\psi(f(t, x, v))) \mathrm{d} \mu(t)(x, v) \mathrm{d} t,
\end{aligned}
$$

where $\mu_{N_{k}}$ and $f_{N_{k}}$ are the elements of the subsequences of the statement of Theorem 4.4.

Proof. Consider a ball $B\left(0, R_{T}\right) \subset \mathbb{R}^{2 d}$, for $R_{T}>0$, so that $\mu_{N_{k}}(t)$ and $\mu(t)$ are compactly supported within $B\left(0, R_{T}\right)$ for all $t \in[0, T]$. Then, since $\mathcal{W}_{1}\left(\mu_{N_{k}}(t), \mu(t)\right) \rightarrow 0$ uniformly in $t \in[0, T]$ for $k \rightarrow \infty$, by assumption (L) we get that $L\left(x, v, \mu_{N_{k}}(t)\right) \rightrightarrows L(x, v, \mu(t))$ uniformly in $(x, v) \in B\left(0, R_{T}\right)$ and $t \in[0, T]$. It follows that

$$
\begin{gathered}
\lim _{k \rightarrow \infty}\left|\int_{0}^{T} \int_{\mathbb{R}^{2 d}}\left[L\left(x, v, \mu_{N_{k}}(t)\right)-L(x, v, \mu(t))\right] \mathrm{d} \mu_{N_{k}}(t)(x, v) \mathrm{d} t\right|= \\
\lim _{k \rightarrow \infty}\left|\int_{0}^{T} \int_{B\left(0, R_{T}\right)}\left[L\left(x, v, \mu_{N_{k}}(t)\right)-L(x, v, \mu(t)]\right) \mathrm{d} \mu_{N_{k}}(t)(x, v) \mathrm{d} t\right|=0 .
\end{gathered}
$$

By Wasserstein convergence and condition (L) we also get for all $t \in[0, T]$

$$
\int_{B\left(0, R_{T}\right)} L(x, v, \mu(t)) \mathrm{d} \mu_{N_{k}}(t)(x, v) \rightarrow \int_{B\left(0, R_{T}\right)} L(x, v, \mu(t)) \mathrm{d} \mu(t)(x, v) .
$$

Since $\mu:[0, T] \rightarrow \mathcal{P}_{1}\left(\mathbb{R}^{2 d}\right)$ is continuous, therefore its image is a compact subset of $P_{1}\left(\mathbb{R}^{2 d}\right)$, again by $(\mathrm{L})$ we get that $L(x, v, \mu(t))$ is uniformly bounded in $B\left(0, R_{T}\right) \times[0, T]$. By dominated convergence this implies

$$
\lim _{k \rightarrow \infty} \int_{0}^{T} \int_{B\left(0, R_{T}\right)} L(x, v, \mu(t)) \mathrm{d} \mu_{N_{k}}(t)(x, v)=\int_{0}^{T} \int_{B\left(0, R_{T}\right)} L(x, v, \mu(t)) \mathrm{d} \mu(t)(x, v) \mathrm{d} t .
$$

Being $\mu_{N_{k}}(t)$ and $\mu(t)$ compactly supported within $B\left(0, R_{T}\right)$ for all $t \in[0, T]$, this is equivalent to

$$
\lim _{k \rightarrow \infty} \int_{0}^{T} \int_{\mathbb{R}^{2 d}} L(x, v, \mu(t)) \mathrm{d} \mu_{N_{k}}(t)(x, v)=\int_{0}^{T} \int_{\mathbb{R}^{2 d}} L(x, v, \mu(t)) \mathrm{d} \mu(t)(x, v) \mathrm{d} t,
$$

which eventually, together with (4.15), gives

$$
\lim _{k \rightarrow \infty} \int_{0}^{T} \int_{\mathbb{R}^{2 d}} L\left(x, v, \mu_{N_{k}}(t)\right) \mathrm{d} \mu_{N_{k}}(t)(x, v) \mathrm{d} t=\int_{0}^{T} \int_{\mathbb{R}^{2 d}} L(x, v, \mu) \mathrm{d} \mu(t)(x, v) \mathrm{d} t .
$$


Since $\mu_{N_{k}}(t)$ are equi-compactly supported, by this uniform convergence and Theorem 2.12 we get the lowersemicontinuity of the second term:

$$
\liminf _{k \rightarrow \infty} \int_{0}^{T} \int_{\mathbb{R}^{2 d}} \psi\left(f_{N_{k}}(t, x, v)\right) \mathrm{d} \mu_{N_{k}}(t)(x, v) \mathrm{d} t \geq \int_{0}^{T} \int_{\mathbb{R}^{2 d}} \psi(f(t, x, v)) \mathrm{d} \mu(t)(x, v) \mathrm{d} t .
$$

By combining (4.16) and (4.17), we eventually show (4.14).

\subsection{Existence of solutions}

With very similar arguments as the ones we used to prove Theorem 4.4 and Corollary 4.6 we obtain the following existence result, with additional limit property of the cost functional. This can be seen as a $\Gamma$-limsup condition.

Theorem 4.7. Assume that we are given maps $H, L$, and $\psi$ as in assumptions $(H),(L)$, and $(\Psi)$ of Section 3. For $1 \leq q<+\infty$ so that (3.2) holds, let $\ell(t)$ be a fixed function in $L^{q}(0, T)$. Let $\mu^{0} \in \mathcal{P}_{1}\left(\mathbb{R}^{2 d}\right)$ be a given probability measure with compact support and $f \in \mathcal{F}_{\ell}$ a forcing term. We assume that the sequence $\left(\mu_{N}^{0}\right)_{N \in \mathbb{N}}$ of atomic empirical measures $\mu_{N}^{0}=\frac{1}{N} \sum_{i=1}^{N} \delta_{\left(\left(x_{N}^{0}\right)_{i},\left(v_{N}^{0}\right)_{i}\right)}(x, v)$ is such that $\lim _{N \rightarrow \infty} \mathcal{W}_{1}\left(\mu_{N}^{0}, \mu^{0}\right)=0$. Let

$$
\mu_{N}(t)(x, v)=\frac{1}{N} \sum_{j=1}^{N} \delta_{\left(x_{i}(t), v_{i}(t)\right)}(x, v),
$$

be supported on the phase space trajectories $\left(x_{i}(t), v_{i}(t)\right) \in \mathbb{R}^{2 d}$, for $i=1, \ldots N$, defining the solution of the system

$$
\left\{\begin{array}{l}
\dot{x}_{i}=v_{i} \\
\dot{v}_{i}=\left(H \star \mu_{N}\right)\left(x_{i}, v_{i}\right)+f\left(t, x_{i}, v_{i}\right), i=1, \ldots N, \quad t \in[0, T]
\end{array}\right.
$$

with initial datum $(x(0), v(0))=\left(x_{N}^{0}, v_{N}^{0}\right)$. Then there exists a map $\mu:[0, T] \rightarrow \mathcal{P}_{1}\left(\mathbb{R}^{2 d}\right)$ such that

(i) $\lim _{N \rightarrow \infty} \mathcal{W}_{1}\left(\mu_{N}(t), \mu(t)\right)=0$ uniformly with respect to $t \in[0, T]$;

(ii) $\mu$ is a weak equi-compactly supported solution of (4.1) with forcing term $f$ in the sense of Definition 4.1;

(iii) the following limit holds:

$$
\begin{aligned}
\lim _{N \rightarrow \infty} & \int_{0}^{T} \int_{\mathbb{R}^{2 d}}\left(L\left(x, v, \mu_{N}(t)\right)+\psi(f(t, x, v))\right) \mathrm{d} \mu_{N}(t)(x, v) \\
= & \int_{0}^{T} \int_{\mathbb{R}^{2 d}}(L(x, v, \mu)+\psi(f(t, x, v))) \mathrm{d} \mu(t)(x, v) \mathrm{d} t .
\end{aligned}
$$

Proof. The results (i) and (ii) can be shown precisely as done in the proof of Theorem 4.4. The only additional note is that, due to Theorem A.8 in Appendix, $\mu$ is actually the unique weak solution of (4.1), hence the whole sequence $\left(\mu_{N}\right)_{N \in \mathbb{N}}$ converges to $\mu$ and not only a subsequence. By uniform convergence of $\mu_{N}$ to $\mu$ in 1 -Wasserstein distance, and by $(2.21)$ with $f_{k}$ constantly equal to $f$ we have

$$
\lim _{N \rightarrow \infty} \int_{0}^{T} \int_{\mathbb{R}^{2 d}} \psi(f(t, x, v)) \mathrm{d} \mu_{N}(t)(x, v)=\int_{0}^{T} \int_{\mathbb{R}^{2 d}} \psi(f(t, x, v)) \mathrm{d} \mu(t)(x, v) \mathrm{d} t
$$

while, arguing as in the proof of (4.16), we have

$$
\lim _{N \rightarrow \infty} \int_{0}^{T} \int_{\mathbb{R}^{2 d}} L\left(x, v, \mu_{N}(t)\right) \mathrm{d} \mu_{N}(t)(x, v) \mathrm{d} t=\int_{0}^{T} \int_{\mathbb{R}^{2 d}} L(x, v, \mu(t)) \mathrm{d} \mu(t)(x, v) \mathrm{d} t .
$$

The limit (4.20) follows. 


\section{Mean-Field optimal control}

We are now able to state the main result of this paper, which is summarizing all the findings we obtained so far, in particular combining the concepts of mean-field and $\Gamma$-limits.

Theorem 5.1. Assume that we are given maps $H, L$, and $\psi$ as in assumptions $(H),(L)$, and $(\Psi)$ of Section 3. For $1 \leq q<+\infty$ so that (3.2) holds, let $\ell(t)$ be a fixed function in $L^{q}(0, T)$. For $N \in \mathbb{N}$ and an initial datum $\left(\left(x_{N}^{0}\right)_{1}, \ldots,\left(x_{N}^{0}\right)_{N},\left(v_{N}^{0}\right)_{1}, \ldots,\left(v_{N}^{0}\right)_{N}\right) \in B\left(0, R_{0}\right) \subset\left(\mathbb{R}^{d}\right)^{N} \times\left(\mathbb{R}^{d}\right)^{N}$, for $R_{0}>0$ independent of $N$, we consider the following finite dimensional optimal control problem

$$
\min _{f \in \mathcal{F}_{\ell}} \int_{0}^{T} \int_{\mathbb{R}^{2 d}}\left[L\left(x, v, \mu_{N}(t)\right)+\psi(f(t, x, v))\right] \mathrm{d} \mu_{N}(t)(x, v) \mathrm{d} t
$$

where

$$
\mu_{N}(t)(x, v)=\frac{1}{N} \sum_{j=1}^{N} \delta_{\left(x_{i}(t), v_{i}(t)\right)}(x, v),
$$

is the time dependent empirical atomic measure supported on the phase space trajectories $\left(x_{i}(t), v_{i}(t)\right) \in \mathbb{R}^{2 d}$, for $i=1, \ldots N$, constrained by being the solution of the system

$$
\left\{\begin{array}{l}
\dot{x}_{i}=v_{i} \\
\dot{v}_{i}=\left(H \star \mu_{N}\right)\left(x_{i}, v_{i}\right)+f\left(t, x_{i}, v_{i}\right), i=1, \ldots, N, \quad t \in[0, T]
\end{array}\right.
$$

with initial datum $(x(0), v(0))=\left(x_{N}^{0}, v_{N}^{0}\right)$ and, for consistency, we set

$$
\mu_{N}^{0}=\frac{1}{N} \sum_{i=1}^{M} \delta_{\left(\left(x_{N}^{0}\right)_{i},\left(v_{N}^{0}\right)_{i}\right)}(x, v) .
$$

For all $N \in \mathbb{N}$ let us denote the function $f_{N} \in \mathcal{F}_{\ell}$ as a solution of the finite dimensional optimal control problem (5.1) and (5.2).

If there exists a compactly supported $\mu_{0} \in \mathcal{P}_{1}\left(\mathbb{R}^{2 d}\right)$ such that $\lim _{N \rightarrow \infty} \mathcal{W}_{1}\left(\mu_{N}^{0}, \mu^{0}\right)=0$, then there exists a subsequence $\left(f_{N_{k}}\right)_{k \in \mathbb{N}}$ and a function $f_{\infty} \in \mathcal{F}_{\ell}$ such that $f_{N_{k}}$ converges to $f_{\infty}$ in the sense of $(2.7)$ and $f_{\infty}$ is a solution of the infinite dimensional optimal control problem

$$
\min _{f \in \mathcal{F}_{\ell}} \int_{0}^{T} \int_{\mathbb{R}^{2 d}}[L(x, v, \mu(t))+\psi(f(t, x, v))] \mathrm{d} \mu(t)(x, v) \mathrm{d} t
$$

where $\mu:[0, T] \rightarrow \mathcal{P}_{1}\left(\mathbb{R}^{2 d}\right)$ is the unique weak solution of

$$
\frac{\partial \mu}{\partial t}+v \cdot \nabla_{x} \mu=\nabla_{v} \cdot[(H \star \mu+f) \mu]
$$

with initial datum $\mu(0):=\mu^{0}$ and forcing term $f$, in the sense of Definition 4.1.

Proof. Let us first of all notice that the existence of an optimal control $f_{N}$ for the finite dimensional optimal control problem (5.1) and (5.2) is ensured by Theorem 3.3. Let $g$ an arbitrary function in $\mathcal{F}_{\ell}$ and $\mu_{g}$ be the corresponding solution to (5.4) with datum $\mu_{g}(0):=\mu^{0}$, which exists thanks to Theorem 4.7 and whose uniqueness follows from Theorem A.8 in Appendix. We also fix the sequence $\left(\mu_{g}\right)_{N}$ of atomic measures uniformly converging to $\mu_{g}$ as in Theorem 4.7. We consider now the converging subsequence $\left(f_{N_{k}}\right)_{k \in \mathbb{N}}$ considered in Theorem 4.4 and we denote $f_{\infty}$ its limit in the sense of (2.7). We further denote with $\mu_{\infty}$ the corresponding 
solution to (5.4), when the forcing term is $f_{\infty}$. Then, by lower-semicontinuity as in Corollary 4.6 and minimality of $f_{N_{k}}$

$$
\begin{aligned}
& \int_{0}^{T} \int_{\mathbb{R}^{2 d}}\left(L\left(x, v, \mu_{\infty}(t)\right)+\psi\left(f_{\infty}(t, x, v)\right)\right) \mathrm{d} \mu_{\infty}(t)(x, v) \mathrm{d} t \\
\leq & \liminf _{k \rightarrow \infty} \int_{0}^{T} \int_{\mathbb{R}^{2 d}}\left(L\left(x, v, \mu_{N_{k}}(t)\right)+\psi\left(f_{N_{k}}(t, x, v)\right)\right) \mathrm{d} \mu_{N_{k}}(t)(x, v) \mathrm{d} t \\
\leq & \liminf _{k \rightarrow \infty} \int_{0}^{T} \int_{\mathbb{R}^{2 d}}\left(L\left(x, v,\left(\mu_{g}\right)_{N_{k}}(t)\right)+\psi(g(t, x, v))\right) d\left(\mu_{g}\right)_{N_{k}}(t)(x, v) \mathrm{d} t \\
= & \int_{0}^{T} \int_{\mathbb{R}^{2 d}}\left(L\left(x, v, \mu_{g}(t)\right)+\psi(g(t, x, v))\right) \mathrm{d} \mu_{g}(t)(x, v) \mathrm{d} t,
\end{aligned}
$$

where the last equality follows from (4.20) in Theorem 4.7. By arbitrariness of $g$, we conclude that $f_{\infty}$ in an optimal control for the problem (5.3) and (5.4).

It is worth mentioning that the main result of Theorem 5.1 can be rephrased as a $\Gamma$-convergence result for the control functionals. Indeed, if we set for any $f \in \mathcal{F}_{\ell}([0, T])$

$$
\mathcal{E}_{\psi, \mu_{N}^{0}}^{N}(f):=\int_{0}^{T} \int_{\mathbb{R}^{2 d}}\left(L\left(x, v, \mu_{N}(t)\right)+\psi(f(t, x, v)) \mathrm{d} \mu_{N}(t)(x, v) \mathrm{d} t,\right.
$$

with $\mu_{N}$ given by (3.8) in correspondence to $f$ and an equi-compactly supported sequence of initial data $\mu_{N}^{0}$, and

$$
\mathcal{E}_{\psi, \mu^{0}}(f):=\int_{0}^{T} \int_{\mathbb{R}^{2 d}}(L(x, v, \mu(t))+\psi(f(t, x, v)) \mathrm{d} \mu(t)(x, v) \mathrm{d} t,
$$

with $\mu$ the unique weak equi-compactly supported solution of (4.1) with initial datum $\mu^{0}$, then (4.14) and (4.20) can be seen respectively as the $\Gamma$-liminf and the $\Gamma$-limsup inequality proving the $\Gamma$-convergence of $\mathcal{E}_{\psi, \mu_{N}^{0}}^{N}$ to $\mathcal{E}_{\psi, \mu^{0}}$ with respect to the weak topology of $L^{q}\left((0, T), W_{l o c}^{1, \infty}\left(\mathbb{R}^{2 d}, \mathbb{R}^{d}\right)\right)$. In particular, Theorem 5.1 can be seen as a consequence of this convergence and of the compactness of the class $\mathcal{F}_{\ell}([0, T])$. Here, it is understood that the functionals are extended to $+\infty$ outside of the class of admissible controls $\mathcal{F}_{\ell}([0, T])$. Notice that actually $(4.20)$ implies that $\mathcal{E}_{\psi, \mu_{N}^{0}}^{N}(f)$ is also pointwise converging to $\mathcal{E}_{\psi, \mu^{0}}(f)$ for any $f \in \mathcal{F}_{\ell}([0, T])$, hence the recovery sequence is in this case simply given by the constant one.

Theorem 5.2. Assume that we are given maps $H, L$, and $\psi$ as in assumptions $(H),(L)$, and $(\Psi)$ of Section 3. For $1 \leq q<+\infty$ so that (3.2) holds, let $\ell(t)$ be a fixed function in $L^{q}(0, T)$, and accordingly define the class $\mathcal{F}_{\ell}([0, T])$ as in Definition 2.1. Consider the functionals $\mathcal{E}_{\psi, \mu_{N}^{0}}^{N}$ and $\mathcal{E}_{\psi, \mu^{0}}$ on $L^{q}\left((0, T), W_{\text {loc }}^{1, \infty}\left(\mathbb{R}^{2 d}, \mathbb{R}^{d}\right)\right)$ with values in $(-\infty,+\infty]$ defined as in (5.5), and (5.6), respectively, when $f \in \mathcal{F}_{\ell}([0, T])$, and equal to $+\infty$ otherwise in $L^{q}\left((0, T), W_{\text {loc }}^{1, \infty}\left(\mathbb{R}^{2 d}, \mathbb{R}^{d}\right)\right)$. Assume also that $\mathcal{W}_{1}\left(\mu_{N}^{0}, \mu^{0}\right) \rightarrow 0$ as $N \rightarrow+\infty$.

Then $\mathcal{E}_{\psi, \mu_{N}^{0}}^{N} \Gamma$-converges to $\mathcal{E}_{\psi, \mu^{0}}$ with respect to the weak topology of $L^{q}\left((0, T), W_{\text {loc }}^{1, \infty}\left(\mathbb{R}^{2 d}, \mathbb{R}^{d}\right)\right.$.

Proof. If $\mathcal{E}_{\psi, \mu^{0}}(f)<+\infty$, that is when $f \in \mathcal{F}_{\ell}([0, T])$, the $\Gamma$-limsup inequality simply follows from the pointwise convergence $\mathcal{E}_{\psi, \mu_{N}^{0}}^{N}(f) \rightarrow \mathcal{E}_{\psi, \mu^{0}}(f)$ proved in $(4.20)$.

Now, assume that $f_{N} \rightarrow f$ weakly in $L^{q}\left((0, T), W_{l o c}^{1, \infty}\left(\mathbb{R}^{2 d}, \mathbb{R}^{d}\right)\right)$. If $\lim \inf _{N \rightarrow+\infty} \mathcal{E}_{\psi, \mu_{N}^{0}}^{N}\left(f_{N}\right)<+\infty$, then $f_{N} \in \mathcal{F}_{\ell}([0, T])$ for $N$ large enough. By the compactness property of Corollary 2.7 and since the limit is uniquely determined, so that we do not need to take subsequences, we deduce $f \in \mathcal{F}_{\ell}([0, T])$ and $f_{N} \rightarrow f$ in the sense of (2.7). By Theorem 4.4, again with no need of taking subsequences since the limit is uniquely determined, we also deduce $\mathcal{W}_{1}\left(\mu_{N}(t), \mu(t)\right) \rightarrow 0$ as $N \rightarrow+\infty$ uniformly in $t \in[0, T]$ so that the $\Gamma$-liminf inequality

$$
\liminf _{N \rightarrow+\infty} \mathcal{E}_{\psi, \mu_{N}^{0}}^{N}\left(f_{N}\right) \geq \mathcal{E}_{\psi, \mu^{0}}(f)
$$

follows by (4.14). 


\section{Appendix A.}

For the reader's convenience we start by briefly recalling some well-known results about solutions to Carathéodory differential equations. We fix an interval $[0, T]$ on the real line, and let $n \geq 1$. Given a domain $\Omega \subset \mathbb{R}^{n}$, a Carathéodory function $g:[0, T] \times \Omega \rightarrow \mathbb{R}^{n}$, and $0<\tau \leq T$, a function $y:[0, \tau] \rightarrow \Omega$ is called a solution of the Carathéodory differential equation

$$
\dot{y}(t)=g(t, y(t))
$$

on $[0, \tau]$ if and only if $y$ is absolutely continuous and (A.1) is satisfied a.e. in $[0, \tau]$. The following existence and uniqueness result holds.

Theorem A.1. Consider an interval $[0, T]$ on the real line, a domain $\Omega \subset \mathbb{R}^{n}, n \geq 1$, and a Carathéodory function $g:[0, T] \times \Omega \rightarrow \mathbb{R}^{n}$. Assume that there exists a function $m \in L^{1}((0, T))$ such that

$$
|g(t, y)| \leq m(t)
$$

for a.e. $t \in[0, T]$ and every $y \in \Omega$. Then, given $y_{0} \in \Omega$, there exists $0<\tau \leq T$ and a solution $y(t)$ of (A.1) on $[0, \tau]$ satisfying $y(0)=y_{0}$.

If in addition there exists a function $l \in L^{1}((0, T))$ such that

$$
\left|g\left(t, y_{1}\right)-g\left(t, y_{2}\right)\right| \leq l(t)\left|y_{1}-y_{2}\right|
$$

for a.e. $t \in[0, T]$ and every $y_{1}, y_{2} \in \Omega$, the solution is uniquely determined on $[0, \tau]$ by the initial condition $y_{0}$.

Proof. See, for instance, ([32], Chapter 1, Thms. 1 and 2).

Also the global existence theorem and a Gronwall estimate on the solutions can be easily generalized to this setting.

Theorem A.2. Consider an interval $[0, T]$ on the real line and a Carathéodory function $g:[0, T] \times \mathbb{R}^{n} \rightarrow \mathbb{R}^{n}$. Assume that there exists a function $m \in L^{1}((0, T))$ such that

$$
|g(t, y)| \leq m(t)(1+|y|)
$$

for a.e. $t \in[0, T]$ and every $y \in \mathbb{R}^{n}$. Then, given $y_{0} \in \mathbb{R}^{n}$, there exists a solution $y(t)$ of (A.1) defined on the whole interval $[0, T]$ which satisfies $y(0)=y_{0}$. Any solution satisfies

$$
|y(t)| \leq\left(\left|y_{0}\right|+\int_{0}^{t} m(s) \mathrm{d} s\right) \mathrm{e}^{\int_{0}^{t} m(s) \mathrm{d} s}
$$

for every $t \in[0, T]$.

If in addition, for every relatively compact open subset of $\mathbb{R}^{n},(\mathrm{~A} .2)$ holds, the solution is uniquely determined on $[0, T]$ by the initial condition $y_{0}$.

Proof. Let $C_{0}:=\left(\left|y_{0}\right|+\int_{0}^{T} m(s) \mathrm{d} s\right) \mathrm{e}^{\int_{0}^{T} m(s) \mathrm{d} s}$. Take a ball $\Omega \subset \mathbb{R}^{p}$ centered at 0 with radius strictly greater than $C_{0}$. Existence of a local solution defined on an interval $[0, \tau]$ and taking values in $\Omega$ follows now easily from (A.3) and Theorem A.1. Using (A.3), any solution of (A.1) with initial datum $y_{0}$ satisfies

$$
|y(t)| \leq\left|y_{0}\right|+\int_{0}^{t} m(s) \mathrm{d} s+\int_{0}^{t} m(s)|y(s)| \mathrm{d} s
$$

for every $t \in[0, \tau]$, therefore (A.4) follows from Gronwall's Lemma. In particular the graph of a solution $y(t)$ cannot reach the boundary of $[0, T] \times \Omega$ unless $\tau=T$, therefore existence of a global solution follows for instance from ([32], Chapter 1, Thm. 4). If (A.2) holds, uniqueness of the global solution follows from Theorem A.1. 
The usual results on continuous dependence on the data hold also in this setting: in particular, we will use this Lemma, following from (A.4) and the Gronwall inequality.

Lemma A.3. Let $g_{1}$ and $g_{2}:[0, T] \times \mathbb{R}^{n} \rightarrow \mathbb{R}^{n}$ be Carathéodory functions both satisfying (A.3) for a function $m \in L^{1}(0, T)$. Let $r>0$ and define

$$
\rho_{r, m, T}:=\left(r+\int_{0}^{T} m(s) \mathrm{d} s\right) \mathrm{e}^{\int_{0}^{T} m(s) \mathrm{d} s} .
$$

Assume in addition that there exists a function $l \in L^{1}(0, T)$

$$
\left|g_{1}\left(t, y_{1}\right)-g_{1}\left(t, y_{2}\right)\right| \leq l(t)\left|y_{1}-y_{2}\right|
$$

for every $t \in[0, T]$ and every $y_{1}, y_{2}$ such that $\left|y_{i}\right| \leq \rho_{r, m, T}, i=1,2$. Set

$$
q(t):=\left\|g_{1}(t, \cdot)-g_{2}(t, \cdot)\right\|_{L^{\infty}\left(B\left(0, \rho_{r, m, T}\right)\right)} .
$$

Then, if $\dot{y}_{1}(t)=g\left(t, y_{1}(t)\right), \dot{y}_{2}(t)=g_{2}\left(t, y_{2}(t)\right),\left|y_{1}(0)\right| \leq r$ and $\left|y_{2}(0)\right| \leq r$, one has

$$
\left|y_{1}(t)-y_{2}(t)\right| \leq \mathrm{e}^{\int_{0}^{t} l(s) \mathrm{d} s}\left|y_{1}(0)-y_{2}(0)\right|+\int_{0}^{t} \mathrm{e}^{\int_{s}^{t} l(\sigma) d \sigma} q(s) \mathrm{d} s
$$

for every $t \in[0, T]$.

We will need the following Lemma. In its statement, we recall that $\mathcal{P}_{1}\left(\mathbb{R}^{n}\right)$ denotes the space of probability measures on $\mathbb{R}^{n}$ with finite first moment. This is a metric space when endowed with the Wasserstein distance $\mathcal{W}_{1}$.

Lemma A.4. Let $H: \mathbb{R}^{n} \rightarrow \mathbb{R}^{p}, n \geq p \geq 1$ be a locally Lipschitz function such that

$$
|H(y)| \leq C(1+|y|), \quad \text { for all } y \in \mathbb{R}^{n},
$$

and $\mu:[0, T] \rightarrow \mathcal{P}_{1}\left(\mathbb{R}^{n}\right)$ be a continuous map with respect to $\mathcal{W}_{1}$. Then there exists a constant $C^{\prime}$ such that

$$
|H \star \mu(t)(y)| \leq C^{\prime}(1+|y|),
$$

for every $t \in[0, T]$ and every $y \in \mathbb{R}^{n}$. Furthermore, if

$$
\operatorname{supp} \mu(t) \subset B(0, R),
$$

for every $t \in[0, T]$, then for every compact subset $K$ of $\mathbb{R}^{n}$ there exists a constant $L_{R, K}$ such that

$$
\left|H \star \mu(t)\left(y_{1}\right)-H \star \mu(t)\left(y_{2}\right)\right| \leq L_{R, K}\left|y_{1}-y_{2}\right|,
$$

for every $t \in[0, T]$ and every $y_{1}, y_{2} \in K$.

Proof. One has from (A.6)

$$
|H \star \mu(t)(y)|=\left|\int_{\mathbb{R}^{n}} H(y-\xi) \mathrm{d} \mu(t)(\xi)\right| \leq C(1+|y|)+C \int_{\mathbb{R}^{n}}|\xi| \mathrm{d} \mu(t)(\xi) ;
$$

since $\int_{\mathbb{R}^{n}}|\xi| \mathrm{d} \mu(t)(\xi)$ is uniformly bounded on $[0, T]$ by our continuity assumption, (A.7) follows.

If (A.8) holds, then for every $y_{1}, y_{2} \in K$ one has

$$
\left|H \star \mu(t)\left(y_{1}\right)-H \star \mu(t)\left(y_{2}\right)\right| \leq \int_{B(0, R)}\left|H\left(y_{1}-\xi\right)-H\left(y_{2}-\xi\right)\right| \mathrm{d} \mu(t)(\xi) \leq L_{R, K}\left|y_{1}-y_{2}\right| .
$$


We now fix a dimension $d \geq 1$ and consider the system of ODE's on $\mathbb{R}^{2 d}$

$$
\left\{\begin{array}{l}
\dot{X}(t)=V(t) \\
\dot{V}(t)=H \star \mu(t)(X(t), V(t))+f(t, X(t), V(t))
\end{array}\right.
$$

on an interval $[0, T]$. Here $X, V$ are both mappings from $[0, T]$ to $\mathbb{R}^{d}, H: \mathbb{R}^{2 d} \rightarrow \mathbb{R}^{d}$ is a locally Lipschitz function satisfying (3.1), $\mu:[0, T] \rightarrow \mathcal{P}_{1}\left(\mathbb{R}^{n}\right)$ is a continuous map with respect to $\mathcal{W}_{1}$ satisfying (A.8) and $f$ belongs to the class $\mathcal{F}_{\ell}$ defined in $(2.22)$ for a fixed function $\ell \in L^{1}(0, T)$. In particular, we have

$$
|f(t, X, V)| \leq \ell(t)(1+|(X, V)|)
$$

for every $V \in \mathbb{R}^{d}$. It follows then from these assumptions and Lemma A.4 that all the hypothesis of Theorem A.2 are satisfied. Therefore, however given $P_{0}:=\left(X_{0}, V_{0}\right)$ in $\mathbb{R}^{2 d}$ there exists a unique solution $P(t):=(X(t), V(t))$ to (A.10) with initial datum $P_{0}$ defined on the whole interval $[0, T]$. We can therefore consider the family of flow maps $\mathcal{T}_{t}^{\mu}: \mathbb{R}^{2 d} \rightarrow \mathbb{R}^{2 d}$ indexed by $t \in[0, T]$ and defined by

$$
\mathcal{T}_{t}^{\mu}\left(P_{0}\right):=P(t)
$$

where $P(t)$ is the value of the unique solution to (A.10) starting from $P_{0}$ at time $t=0$. The notation aims also at stressing the dependence of these flow maps on the given mapping $\mu(t)$. We can easily recover, as consequence of (A.5), similar estimates as in [10], Lemmas 3.7 and 3.8: we report the statement and a sketch of the proof of this result to allow the reader to keep track of the dependence of these constants on the data of the problem.

Lemma A.5. Let $H: \mathbb{R}^{2 d} \rightarrow \mathbb{R}^{d}$ be a locally Lipschitz function satisfying (3.1), let $\mu:[0, T] \rightarrow \mathcal{P}_{1}\left(\mathbb{R}^{2 d}\right)$ and $\nu:[0, T] \rightarrow \mathcal{P}_{1}\left(\mathbb{R}^{2 d}\right)$ be continuous maps with respect to $\mathcal{W}_{1}$ both satisfying

$$
\operatorname{supp} \mu(t) \subset B(0, R) \quad \text { and } \quad \operatorname{supp} \nu(t) \subset B(0, R)
$$

for every $t \in[0, T]$. Consider $f$ belonging to the class $\mathcal{F}_{\ell}$ introduced in Definition, for a fixed function $\ell \in$ $L^{1}(0, T)$, and the flow maps $\mathcal{T}_{t}^{\mu}$ and $\mathcal{T}_{t}^{\nu}$ associated to the system (A.10) and to the system

$$
\left\{\begin{array}{l}
\dot{X}(t)=V(t) \\
\dot{V}(t)=H \star \nu(t)(X(t), V(t))+f(t, X(t), V(t)),
\end{array}\right.
$$

respectively, on $[0, T]$. Let $C$ be the constant in (3.1). Fix $r>0$ : then there exist a constant $\rho$ and a function $l \in L^{1}(0, T)$, both depending only on $r, C, R, \ell$, and $T$ such that

$$
\left|\mathcal{T}_{t}^{\mu}\left(P_{1}\right)-\mathcal{T}_{t}^{\nu}\left(P_{2}\right)\right| \leq \mathrm{e}^{\int_{0}^{t} l(s) \mathrm{d} s}\left|P_{1}-P_{2}\right|+\int_{0}^{t} \mathrm{e}^{\int_{s}^{t} l(\sigma) d \sigma}\|H \star \mu(s)-H \star \nu(s)\|_{L^{\infty}(B(0, \rho))} \mathrm{d} s
$$

whenever $\left|P_{1}\right| \leq r$ and $\left|P_{2}\right| \leq r$, for every $t \in[0, T]$.

Proof. Let $g_{1}$ and $g_{2}:[0, T] \times \mathbb{R}^{2 d} \rightarrow \mathbb{R}^{2 d}$ be the right-hand sides of (A.10), and (A.14), respectively. As in (A.7) we can find a constant $C^{\prime}$ which depends only on $C$ and $R$ such that

$$
|H \star \mu(t)(P)| \leq C^{\prime}(1+|P|) \quad \text { and } \quad|H \star \nu(t)(P)| \leq C^{\prime}(1+|P|)
$$

for every $t \in[0, T]$ and every $P \in \mathbb{R}^{2 d}$. Setting now $\hat{m}(t)=1+C^{\prime}+\ell(t)$ and also using (A.11), it follows that $g_{1}$ and $g_{2}$ both satisfy (A.3) with $m(t)$ replaced by $\hat{m}(t)$. Therefore, for every $P_{1}$ and $P_{2} \in \mathbb{R}^{2 d}$ such that $\left|P_{i}\right| \leq r$, $i=1,2$ and every $t \in[0, T],($ A.4) gives

$$
\left|\mathcal{T}_{t}^{\mu}\left(P_{1}\right)\right| \leq\left(r+\int_{0}^{T} \hat{m}(s) \mathrm{d} s\right) \mathrm{e}^{\int_{0}^{T} \hat{m}(s) \mathrm{d} s} \quad \text { and } \quad\left|\mathcal{T}_{t}^{\nu}\left(P_{2}\right)\right| \leq\left(r+\int_{0}^{T} \hat{m}(s) \mathrm{d} s\right) \mathrm{e}^{\int_{0}^{T} \hat{m}(s) \mathrm{d} s} .
$$


Set $\rho:=\left(r+\int_{0}^{T} \hat{m}(s) \mathrm{d} s\right) \mathrm{e}^{\int_{0}^{T} \hat{m}(s) \mathrm{d} s}$. Now, obviously

$$
\left\|g_{1}(t, \cdot)-g_{2}(t, \cdot)\right\|_{L^{\infty}(B(0, \rho))}=\|H \star \mu(t)-H \star \nu(t)\|_{L^{\infty}(B(0, \rho))}
$$

for every $t \in[0, T]$. Furthermore, by (A.9), the definition of $\rho$, and since $f$ belongs to $\mathcal{F}_{\ell}$, the Lipschitz constant of $g_{1}(t, \cdot)$ on $B(0, \rho)$ can be estimated for a.e. $t \in[0, T]$ with a function $l(t) \in L^{1}(0, T)$ only depending on $\ell(t)$, $R, C, r$ and $T$. With this, the conclusion follows at once from (A.5).

We will use (4.6) to prove uniqueness and stability of equi-compactly supported solutions of (4.3). We recall the following two Lemmata, both proved in [10].

Lemma A.6. Let $E_{1}$ and $E_{2}: \mathbb{R}^{n} \rightarrow \mathbb{R}^{n}$ be two bounded Borel measurable functions. Then, for every $\mu \in$ $\mathcal{P}_{1}\left(\mathbb{R}^{n}\right)$ one has

$$
\mathcal{W}_{1}\left(\left(E_{1}\right)_{\sharp} \mu,\left(E_{2}\right)_{\sharp} \mu\right) \leq\left\|E_{1}-E_{2}\right\|_{L^{\infty}(\operatorname{supp} \mu)} .
$$

If in addition $E_{1}$ is locally Lipschitz continuous, and $\mu, \nu \in \mathcal{P}_{1}\left(\mathbb{R}^{n}\right)$ are both compactly supported on a ball $B_{r}$ of $\mathbb{R}^{n}$, then

$$
\mathcal{W}_{1}\left(\left(E_{1}\right)_{\sharp} \mu,\left(E_{1}\right)_{\sharp} \nu\right) \leq L_{r} \mathcal{W}_{1}(\mu, \nu),
$$

where $L_{r}$ is the Lipschitz constant of $E_{1}$ on $B_{r}$.

Proof. For the sake of the reader, we sketch only the proof of (A.17) as it does not appear exactly equally reported in ([10], Lem. 3.11 and 3.15). Let $\tilde{\pi}$ be the optimal transfer plan for $\left(E_{1}\right)_{\sharp} \mu$ and $\left(E_{1}\right)_{\sharp \nu}$ and $\pi$ the one of $\mu$ and $\nu$. Then

$$
\begin{aligned}
\mathcal{W}_{1}\left(\left(E_{1}\right)_{\sharp} \mu,\left(E_{1}\right)_{\sharp} \nu\right) & =\int_{\mathbb{R}^{n} \times \mathbb{R}^{n}}|x-y| d \tilde{\pi}(x, y) \leq \int_{\mathbb{R}^{n} \times \mathbb{R}^{n}}|x-y| d\left(\left(E_{1} \times E_{1}\right)_{\sharp} \pi\right)(x, y) \\
& =\int_{\mathbb{R}^{n} \times \mathbb{R}^{n}}\left|E_{1}(x)-E_{1}(y)\right| \mathrm{d} \pi(x, y)=L_{r} \int_{\mathbb{R}^{n} \times \mathbb{R}^{n}}|x-y| \mathrm{d} \pi(x, y)=L_{r} \mathcal{W}_{1}(\mu, \nu) .
\end{aligned}
$$

Lemma A.7. Let $H: \mathbb{R}^{2 d} \rightarrow \mathbb{R}^{d}$ be a locally Lipschitz function satisfying (3.1), let $\mu:[0, T] \rightarrow \mathcal{P}_{1}\left(\mathbb{R}^{2 d}\right)$ and $\nu:[0, T] \rightarrow \mathcal{P}_{1}\left(\mathbb{R}^{2 d}\right)$ be continuous maps with respect to $\mathcal{W}_{1}$ both satisfying

$$
\operatorname{supp} \mu(t) \subset B(0, R) \quad \text { and } \quad \operatorname{supp} \nu(t) \subset B(0, R)
$$

for every $t \in[0, T]$. Then for every $\rho>0$ there exists a constant $L_{\varrho, R}$ such that

$$
\|H \star \mu(t)-H \star \nu(t)\|_{L^{\infty}(B(0, \rho))} \leq L_{\varrho, R} \mathcal{W}_{1}(\mu(t), \nu(t))
$$

for every $t \in[0, T]$.

Proof. See Lemma 4.7 from [10].

With the previous Lemmata and (A.15), we can easily prove the following result.

Theorem A.8. Fix a function $f$ belonging to the class $\mathcal{F}_{\ell}$ for a given $\ell \in L^{1}(0, T)$. Consider a locally Lipschitz function $H: \mathbb{R}^{2 d} \rightarrow \mathbb{R}^{d}$ satisfying (3.1) with a constant $C$. Fix $T>0$ and let $\mu(t)$ and $\nu(t)$ be two equi-compactly supported solutions of (4.1) with forcing term $f$ on the interval $[0, T]$. Let $\mu_{0}:=\mu(0)$ and $\nu_{0}:=\nu(0)$. Consider $r>0$ such that

and $R>0$ such that

$$
\operatorname{supp} \mu_{0} \subset B(0, r) \quad \text { and } \quad \operatorname{supp} \nu_{0} \subset B(0, r)
$$

$$
\operatorname{supp} \mu(t) \subset B(0, R) \quad \text { and } \quad \operatorname{supp} \nu(t) \subset B(0, R)
$$


for every $t \in[0, T]$. Then, there exist a function $\delta \in L^{1}(0, T)$ depending only on $r, C, R, \ell$, and $T$ such that

$$
\mathcal{W}_{1}(\mu(t), \nu(t)) \leq \mathrm{e}^{\int_{0}^{t} \delta(s) \mathrm{d} s} \mathcal{W}_{1}\left(\mu_{0}, \nu_{0}\right)
$$

for every $t \in[0, T]$. In particular, equi-compactly supported solutions of (4.3) are uniquely determined by the initial datum.

Proof. Let $\mathcal{T}_{t}^{\mu}$ and $\mathcal{T}_{t}^{\nu}$ be the flow maps associated to the system (A.10) and to the system (A.14), respectively. By (4.6), the triangle inequality, and Lemma A.6 we have for every $t$

$$
\begin{gathered}
\mathcal{W}_{1}(\mu(t), \nu(t))=\mathcal{W}_{1}\left(\left(\mathcal{T}_{t}^{\mu}\right)_{\sharp} \mu_{0},\left(\mathcal{T}_{t}^{\nu}\right)_{\sharp} \nu_{0}\right) \\
\leq \mathcal{W}_{1}\left(\left(\mathcal{T}_{t}^{\mu}\right)_{\sharp} \mu_{0},\left(\mathcal{T}_{t}^{\mu}\right)_{\sharp} \nu_{0}\right)+\mathcal{W}_{1}\left(\left(\mathcal{T}_{t}^{\mu}\right)_{\sharp} \nu_{0},\left(\mathcal{T}_{t}^{\nu}\right)_{\sharp} \nu_{0}\right) \leq L_{r} \mathcal{W}_{1}\left(\mu_{0}, \nu_{0}\right)+\left\|\mathcal{T}_{t}^{\mu}-\mathcal{T}_{t}^{\nu}\right\|_{L^{\infty}(B(0, r))}
\end{gathered}
$$

where $L_{r}$ is the Lipschitz constant of $\mathcal{T}_{t}^{\mu}$ on the ball $B(0, r)$.

Using (A.15) with $\mu=\nu$ we get that there exists a function $l \in L^{1}(0, T)$ only depending on $r, C, R, \ell$, and $T$ such that

$$
L_{r} \leq \mathrm{e}^{\int_{0}^{t} l(s) \mathrm{d} s} .
$$

Again by (A.15) with $P_{1}=P_{2}$ there exist a constant $\rho$ and an $L^{1}$ function, still denoted by $l$, both depending only on $r, C, R, \ell$, and $T$ such that

$$
\left\|\mathcal{T}_{t}^{\mu}-\mathcal{T}_{t}^{\nu}\right\|_{L^{\infty}(B(0, r))} \leq \int_{0}^{t} \mathrm{e}^{\int_{s}^{t} l(\sigma) d \sigma}\|H \star \mu(s)-H \star \nu(s)\|_{L^{\infty}(B(0, \rho))} \mathrm{d} s .
$$

Combining (A.20), (A.21), and (A.22) with Lemma A.7, we get the existence of an $L^{1}$ function, still denoted by $l(t)$, and of a constant $L$, both depending only on $r, C, R, \ell$, and $T$ such that

$$
\mathcal{W}_{1}(\mu(t), \nu(t)) \leq \mathrm{e}^{\int_{0}^{t} l(s) \mathrm{d} s} \mathcal{W}_{1}\left(\mu_{0}, \nu_{0}\right)+L \int_{0}^{t} \mathrm{e}^{\int_{s}^{t} l(\sigma) d \sigma} \mathcal{W}_{1}(\mu(s), \nu(s)) \mathrm{d} s
$$

for every $t \in[0, T]$, or equivalently

$$
\mathrm{e}^{-\int_{0}^{t} l(s) \mathrm{d} s} \mathcal{W}_{1}(\mu(t), \nu(t)) \leq \mathcal{W}_{1}\left(\mu_{0}, \nu_{0}\right)+L \int_{0}^{t} \mathrm{e}^{-\int_{0}^{s} l(\sigma) d \sigma} \mathcal{W}_{1}(\mu(s), \nu(s)) \mathrm{d} s .
$$

The Gronwall inequality gives now

$$
\mathrm{e}^{-\int_{0}^{t} l(s) \mathrm{d} s} \mathcal{W}_{1}(\mu(t), \nu(t)) \leq \mathcal{W}_{1}\left(\mu_{0}, \nu_{0}\right) \mathrm{e}^{L t}
$$

which is exactly (A.19) with $\delta(t)=L+l(t)$.

Remark A.9. The existence result of Theorem 4.7 gives an explicit estimate of an $R$ satisfying (A.18), once the constants $r, C$, and $T$, and the function $\ell$ appearing in the statement of Theorem A.8 are given. As a byproduct of uniqueness, the function $\delta$ in (A.19) is therefore only depending on $r, C$, $\ell$, and $T$.

Acknowledgements. Massimo Fornasier acknowledges the support of the ERC-Starting Grant HDSPCONTR "HighDimensional Sparse Optimal Control". Francesco Solombrino acknowledges the hospitality of the Johann Radon Institute for Computational and Applied Mathematics (RICAM) of the Austrian Academy of Sciences during the early preparation of this work. 


\section{REFERENCES}

[1] N. Ahmed and X. Ding, Controlled McKean-Vlasov equations. Commun. Appl. Anal. 5 (2001) 183-206.

[2] L. Ambrosio, N. Fusco and D. Pallara, Functions of Bounded Variation and Free Discontinuity Problems. Oxford, Clarendon Press (2000).

[3] L. Ambrosio, N. Gigli and G. Savaré, Gradient Flows in Metric Spaces and in the Space of Probability Measures. Lect. Math. ETH Zürich 2nd, edition. Birkhäuser Verlag, Basel (2008).

[4] D. Andersson and B. Djehiche, A maximum principle for SDEs of mean-field type. Appl. Math. Opt. 63 (2011) $341-356$.

[5] M. Ballerini, N. Cabibbo, R. Candelier, A. Cavagna, E. Cisbani, L. Giardina, L. Lecomte, A. Orlandi, G. Parisi, A. Procaccini, M. Viale and V. Zdravkovic, Interaction ruling animal collective behavior depends on topological rather than metric distance: evidence from a field study. Proc. National Academy of Sci. 105 (2008) 1232-1237.

[6] A. Bensoussan, J. Frehse and P. Yam, Mean field games and mean field type control theory. Springer, New York (2013).

[7] A. Bressan and B. Piccoli, Introduction to the Mathematical Theory of Control, vol. 2 of AIMS Ser. Appl. Math.. American Institute of Mathematical Sciences (AIMS), Springfield, MO (2007).

[8] R. Buckdahn, B. Djehiche and J. Li, A general stochastic maximum principle for sdes of mean-field type. Appl. Math. Opt. 64 (2011) 197-216.

[9] S. Camazine, J. Deneubourg, N. Franks, J. Sneyd, G. Theraulaz and E. Bonabeau, Self-Organization in Biological Systems. Princeton University Press (2003).

[10] J.A. Cañizo, J.A. Carrillo and J. Rosado, A well-posedness theory in measures for some kinetic models of collective motion. Math. Model. Meth. Appl. Sci. 21 (2011) 515-539.

[11] M. Caponigro, M. Fornasier, B. Piccoli and E. Trélat, Sparse stabilization and control of the Cucker-Smale model. Preprint: arXiv:1210.5739 (2012).

[12] J.A. Carrillo, Y.-P. Choi and M. Hauray, The derivation of swarming models: mean-field limit and Wasserstein distances. Preprint: arXiv:1304.5776 (2013).

[13] J.A. Carrillo, M.R. D'Orsogna and V. Panferov, Double milling in self-propelled swarms from kinetic theory. Kinet. Relat. Models 2 (2009) 363-378.

[14] J. A. Carrillo, M. Fornasier, G. Toscani and F. Vecil, Particle, kinetic, and hydrodynamic models of swarming, in Math. Modeling of Collective Behavior in Socio-Economic and Life Sci., edited by G. Naldi, L. Pareschi, G. Toscani and N. Bellomo. Model. Simul. Sci. Engrg. Technol. Birkhäuser, Boston (2010) 297-336.

[15] E. Casas, C. Clason and K. Kunisch, Approximation of elliptic control problems in measure spaces with sparse solutions. SIAM J. Control Optim. 50 (2012) 1735-1752.

[16] Y. Chuang, M. D’Orsogna, D. Marthaler, A. Bertozzi and L. Chayes, State transition and the continuum limit for the 2D interacting, self-propelled particle system. Physica D 232 (2007) 33-47.

[17] Y. Chuang, Y. Huang, M. D’Orsogna and A. Bertozzi, Multi-vehicle flocking: scalability of cooperative control algorithms using pairwise potentials. IEEE Int. Conference on Robotics and Automation (2007) 2292-2299.

[18] C. Clason and K. Kunisch, A duality-based approach to elliptic control problems in non-reflexive Banach spaces. ESAIM: COCV 17 (2011) 243-266.

[19] C. Clason and K. Kunisch, A measure space approach to optimal source placement. Comput. Optim. Appl. 53 (2012) $155-171$.

[20] I. Couzin and N. Franks, Self-organized lane formation and optimized traffic flow in army ants. Proc. R. Soc. London B 270 (2002) 139-146.

[21] I. Couzin, J. Krause, N. Franks and S. Levin, Effective leadership and decision making in animal groups on the move. Nature 433 (2005) 513-516.

[22] A. J. Craig and I. Flügge-Lotz, Investigation of optimal control with a minimum-fuel consumption criterion for a fourth-order plant with two control inputs; synthesis of an efficient suboptimal control. J. Basic Engrg. 87 (1965) 39-58.

[23] E. Cristiani, B. Piccoli and A. Tosin, Modeling self-organization in pedestrians and animal groups from macroscopic and microscopic viewpoints, in Mathematical Modeling of Collective Behavior in Socio-Economic and Life Sciences, edited by G. Naldi, L. Pareschi, G. Toscani and N. Bellomo. Model. Simul. Sci. Engrg. Technol. Birkhäuser, Boston (2010).

[24] E. Cristiani, B. Piccoli and A. Tosin, Multiscale modeling of granular flows with application to crowd dynamics. Multiscale Model. Simul. 9 (2011) 155-182.

[25] F. Cucker and J.-G. Dong, A general collision-avoiding flocking framework. IEEE Trans. Automat. Control 56 (2011) 11241129.

[26] F. Cucker and E. Mordecki, Flocking in noisy environments. J. Math. Pures Appl. 89 (2008) 278-296.

[27] F. Cucker and S. Smale, Emergent behavior in flocks. IEEE Trans. Automat. Control 52 (2007) 852-862,

[28] F. Cucker and S. Smale, On the mathematics of emergence. Japan J. Math. 2 (2007) 197-227.

[29] F. Cucker, S. Smale and D. Zhou, Modeling language evolution. Found. Comput. Math. 4 (2004) 315-343.

[30] G. Dal Maso, An Introduction to $\Gamma$-Convergence. Progress in Nonlinear Differ. Eqs. Appl., vol. 8. Birkhäuser Boston Inc., Boston, MA (1993).

[31] H. Federer, Geometric Measure Theory. Die Grundlehren der mathematischen Wissenschaften in Einzeldarstellungen, vol. 153. Springer-Verlag, Berlin, Heidelberg, New York (1969).

[32] A.F. Filippov, Differential equations with Discontinuous Righthand Sides. Vol. 18 of Math. Appl. (Soviet Series). Translated from the Russian. Kluwer Academic Publishers Group, Dordrecht (1988). 
[33] R. Glowinski, Numerical Methods for Nonlinear Variational Problems. Scientific Comput. Springer-Verlag, Berlin (2008). Reprint of the 1984 original.

[34] G. Grégoire and H. Chaté, Onset of collective and cohesive motion. Phys. Rev. Lett. 92 (2004).

[35] R. Herzog, G. Stadler and G. Wachsmuth, Directional sparsity in optimal control of partial differential equations. SIAM J. Control Optim. 50 (2012) 943-963.

[36] M. Huang, P. Caines and R. Malhamé, Individual and mass behaviour in large population stochastic wireless power control problems: centralized and Nash equilibrium solutions. Proc. of the 42nd IEEE Conference on Decision and Control Maui, Hawaii USA (2003) 98-103.

[37] A. Jadbabaie, J. Lin and A.S. Morse, Correction to: "Coordination of groups of mobile autonomous agents using nearest neighbor rules" [48 (2003) 988-1001; MR 1986266]. IEEE Trans. Automat. Control 48 (2003) 1675.

[38] J. Ke, J. Minett, C.-P. Au and W.-Y. Wang, Self-organization and selection in the emergence of vocabulary. Complexity 7 (2002) 41-54.

[39] E. F. Keller and L.A. Segel, Initiation of slime mold aggregation viewed as an instability. J. Theoret. Biol. 26 (1970) $399-415$.

[40] A. Koch and D. White, The social lifestyle of myxobacteria. Bioessays 20 (1998) 1030-1038.

[41] J.-M. Lasry and P.-L. Lions, Mean field games. Japan J. Math. 2 (2007) 229-260.

[42] N. Leonard and E. Fiorelli, Virtual leaders, artificial potentials and coordinated control of groups. Proc. of 40th IEEE Conf. Decision Contr. (2001) 2968-2973.

[43] H. Niwa, Self-organizing dynamic model of fish schooling. J. Theoret. Biol. 171 (1994) 123-136.

[44] M. Nuorian, P. Caines and R. Malhamé, Synthesis of Cucker-Smale type flocking via mean field stochastic control theory: Nash equilibria. Proc. of 48th Allerton Conf. Comm., Cont. Comp., Monticello, Illinois (2010) 814-815.

[45] M. Nuorian, P. Caines and R. Malhamé, Mean field analysis of controlled Cucker-Smale type flocking: Linear analysis and perturbation equations. Proc. of 18th IFAC World Congress Milano, Italy (2011) 4471-4476.

[46] J. Parrish and L. Edelstein-Keshet, Complexity, pattern and evolutionary trade-offs in animal aggregation. Science 294 (1999) 99-101.

[47] J. Parrish, S. Viscido and D. Gruenbaum, Self-organized fish schools: An examination of emergent properties. Biol. Bull. 202 (2002) 296-305.

[48] L. Perea, G. Gómez and P. Elosegui, Extension of the Cucker-Smale control law to space flight formations. AIAA J. Guidance, Control, and Dynamics 322009 527-537.

[49] B. Perthame, Mathematical tools for kinetic equations. Bull. Am. Math. Soc., New Ser. 41 (2004) $205-244$.

[50] B. Perthame, Transport Equations in Biology. Basel, Birkhäuser (2007).

[51] K. Pieper and B. Vexler, A priori error analysis for discretization of sparse elliptic optimal control problems in measure space. SIAM J. Control Optim. 51 (2013) 2788-2808.

[52] Y. Privat, E. Trélat and E. Zuazua, Complexity and regularity of maximal energy domains for the wave equation with fixed initial data. Discrete Contin. Dyn. Syst. Ser. A.

[53] R. Rannacher and B. Vexler, Adaptive finite element discretization in PDE-based optimization. GAMM-Mitt. 33 (2010) 177-193.

[54] W. Romey, Individual differences make a difference in the trajectories of simulated schools of fish. Ecol. Model. 92 (1996) $65-77$.

[55] M.B. Short, M. R. D’Orsogna, V.B. Pasour, G.E. Tita, P.J. Brantingham, A.L. Bertozzi and L.B. Chayes, A statistical model of criminal behavior. Math. Models Methods Appl. Sci. 18 (2008) 1249-1267.

[56] G. Stadler, Elliptic optimal control problems with $L^{1}$-control cost and applications for the placement of control devices. Comput. Optim. Appl. 44 (2009) 159-181.

[57] K. Sugawara and M. Sano, Cooperative acceleration of task performance: Foraging behavior of interacting multi-robots system. Phys. D 100 (1997) 343-354.

[58] J. Toner and Y. Tu, Long-range order in a two-dimensional dynamical xy model: How birds fly together. Phys. Rev. Lett. 75 (1995) 4326-4329.

[59] T. Vicsek, A. Czirok, E. Ben-Jacob, I. Cohen and O. Shochet, Novel type of phase transition in a system of self-driven particles. Phys. Rev. Lett. 75 (1995) 1226-1229.

[60] T. Vicsek and A. Zafeiris, Collective motion. Phys. Rep. 517 (2012) 71-140.

[61] C. Villani, Optimal Transport, vol. 338. Grundlehren der Math. Wissenschaften, [Fundamental Principles of Mathematical Science]. Springer-Verlag, Berlin (2009). Old and new.

[62] G. Vossen and H. Maurer, $L^{1}$ minimization in optimal control and applications to robotics. Optim. Control Appl. Methods 27 (2006) 301-321.

[63] G. Wachsmuth and D. Wachsmuth, Convergence and regularization results for optimal control problems with sparsity functional. ESAIM: COCV 17 (2011) 858-886.

[64] C. Yates, R. Erban, C. Escudero, L. Couzin, J. Buhl, L. Kevrekidis, P. Maini and D. Sumpter, Inherent noise can facilitate coherence in collective swarm motion. Proc. Natl. Acad. Sci. 106 (2009) 5464-5469. 